\title{
Magnetic deformation of super-Maxwell theory in supergravity
}

\author{
Ignatios Antoniadis, ${ }^{a, b}$ Jean-Pierre Derendinger, ${ }^{a}$ Hongliang Jiang ${ }^{a}$ and \\ Gabriele Tartaglino-Mazzucchelli ${ }^{a, c}$ \\ ${ }^{a}$ Albert Einstein Center, Institute for Theoretical Physics, University of Bern, \\ Sidlerstrasse 5, 3012 Bern, Switzerland \\ ${ }^{b}$ Laboratoire de Physique Théorique et Hautes Energies - LPTHE, Sorbonne Université, CNRS, \\ 4 Place Jussieu, 75005 Paris, France \\ ${ }^{c}$ School of Mathematics and Physics, The University of Queensland, \\ Physics Annexe (building 6), St Lucia, Brisbane, QLD 4072, Australia \\ E-mail: antoniadis@itp.unibe.ch, derendinger@itp.unibe.ch, \\ jiang@itp.unibe.ch, g.tartaglino-mazzucchelli@uq.edu.au
}

ABSTRACT: A necessary condition for partial breaking of $\mathcal{N}=2$ global supersymmetry is the presence of nonlinear deformations of the field transformations which cannot be generated by background values of auxiliary fields. This work studies the simplest of these deformations which already occurs in $\mathcal{N}=1$ global supersymmetry, and its coupling to supergravity. It can be viewed as an imaginary constant shift of the $D$-auxiliary real field of an abelian gauge multiplet. We show how this deformation describes the magnetic dual of a Fayet-Iliopoulos term, a result that remains valid in supergravity, using its new-minimal formulation. Local supersymmetry and the deformation induce a positive cosmological constant. Moreover, the deformed U(1) Maxwell theory coupled to supergravity describes upon elimination of the auxiliary fields the gauging of $R$-symmetry, realised by the Freedman model of 1976. To this end, we construct the chiral spinor multiplet in superconformal tensor calculus by working out explicitly its transformation rules and use it for an alternative description of the new-minimal supergravity coupled to a $\mathrm{U}(1)$ multiplet. We also discuss the deformed Maxwell theory in curved superspace.

KEYWORDS: Supergravity Models, Supersymmetry and Duality, Supersymmetric Effective Theories

ARXIV EPRINT: 2005.11374 


\section{Contents}

1 Introduction 1

$2 \quad D$-deformation in global $\mathcal{N}=1$ supersymmetry 3

$\begin{array}{lll}3 & \text { Supergravity } & 6\end{array}$

$\begin{array}{lll}3.1 & \text { New minimal supergravity } & 7\end{array}$

4 The deformed super-Maxwell theory in supergravity 9

5 On the Fayet-Iliopoulos term in supergravity $\quad 15$

6 On the electric-magnetic duality 18

$\begin{array}{llr}7 & \text { Concluding remarks } & 21\end{array}$

A Conventions and some useful formulae $\quad 23$

B The superconformal chiral spinor multiplet $\quad 24$

B.1 Chiral spinor multiplet in the real field basis and its decomposition 24

$\begin{array}{ll}\text { B.2 Chiral spinor multiplet in chiral multiplet basis } 28 & 28\end{array}$

$\begin{array}{ll}\text { C Deformed Maxwell theory in curved superspace } & 29\end{array}$

\section{Introduction}

Deformations of supersymmetry transformations play an important role for realising a partial breaking of extended supersymmetry [1-3]. In $\mathcal{N}=2$ super-Maxwell theory, such deformations involve six parameters. Three of them can be generated by background values of auxiliary fields of the off-shell representation, in a real SU(2) $R$-symmetry vector. The other three are their magnetic counterparts, absent in off-shell representations. They can be obtained formally by considering a constant imaginary part for every component of the $\mathrm{SU}(2)$ triplet of auxiliary fields. In other words, these six deformation parameters form a complex $\mathrm{SU}(2)$ vector $\vec{Y}$ and global supersymmetry is partially broken $\mathcal{N}=2 \rightarrow \mathcal{N}=1$ if the deformation vector is non-trivial and nilpotent in the vacuum:

$$
|\vec{Y}|^{2}>0, \quad \vec{Y}^{2}=0 .
$$

This is explained for instance in refs. [3, 4].

An interesting question is to understand the coupling of a deformed supersymmetric theory to supergravity in relation to (partial) supersymmetry breaking. In this work, 
we make a first step towards this investigation by studying a non-trivial supersymmetry deformation in a simpler context, namely at the level of $\mathcal{N}=1$. The type of deformation considered in the present paper has already appeared in the context of the supersymmetric Dirac-Born-Infeld theory and partial $\mathcal{N}=2 \rightarrow \mathcal{N}=1$ supersymmetry breaking [4-6] but here we will focus on a purely $\mathcal{N}=1$ analysis.

Indeed, in $\mathcal{N}=1$ super-Maxwell theory, the real auxiliary field $D$ can generate an "electric" deformation (equivalent to a Fayet-Iliopoulos term), while its magnetic counterpart, which can be formally obtained by adding to $D$ a constant imaginary part in the supersymmetry variations, corresponds to an integration constant in the supersymmetric Bianchi identity. In our analysis here, we show that such an integration constant is equivalent to a 'magnetic' Fayet-Iliopoulos term, dual under electric-magnetic (EM) duality to an 'electric' Fayet-Iliopoulos term. A corollary of this result is that one cannot add 'electrically' charged chiral multiplets in a local action containing the deformation, since they would correspond to magnetic monopoles in the dual theory which has a Fayet-Iliopoulos term. In the presence of several U(1)'s with corresponding deformation parameters, charged matter should satisfy the condition of being neutral under the U(1) combination containing the deformation, while non trivial charges can exist with respect to all orthogonal combinations for which supersymmetry variations are not deformed.

We then proceed to the description of the deformation in supergravity, considering the simplest case of pure $\mathcal{N}=1$ supergravity coupled to an abelian $\mathrm{U}(1)$ multiplet. The main observation is that an integration constant in the supersymmetric Bianchi identity can be obtained as a background value of a linear multiplet. Since the Bianchi identity involves the chiral spinor gauge field-strength superfield $W_{\alpha}$, it is natural to consider a general chiral spinor superfield which contains the degrees of freedom of a Maxwell multiplet and of a linear multiplet $[7,8]$. The usual Bianchi identity eliminates the latter and leaves the gauge multiplet in the physical spectrum, while the presence of an integration constant may arise from a background value for the linear multiplet. To avoid adding extra degrees of freedom in the theory, we identify the linear multiplet with the compensator of the newminimal $[9,10]$ off-shell formulation of $\mathcal{N}=1$ supergravity $[7,8,11]$. The deformed Maxwell theory can thus be constructed in a similar way as the undeformed one by implementing the deformation in the Bianchi identity of $W_{\alpha}$ stemmed from the linear compensator.

As in the global case, one finds that the deformation becomes a Fayet-Iliopoulos term in supergravity after performing a EM duality. The Fayet-Iliopoulos term generates a positive cosmological constant proportional to the square of the $\mathrm{U}(1)$ coupling and can be described by the well-known Freedman model where the $R$-symmetry is gauged and the gravitino and gaugino are charged under it [12]. Therefore, the deformed theory provides a magnetic dual description of the Freedman model off-shell. However, after eliminating the auxiliary fields in the supergravity context on the deformed theory side, the leftover propagating $\mathrm{U}(1)$ vector boson gauges again the $R$-symmetry under which the fermions are charged (see e.g. [11, 13]).

More precisely, from the argument in global supersymmetry presented above, one would expect the absence of charged particles on the side of the deformed theory. Indeed the fermions (gravitino and gaugino) are neutral under the off-shell Maxwell field. However, 
one finds that the latter becomes unphysical and upon appropriate gauge conditions and integration over all auxiliary fields, the physical propagating gauge field corresponds now to the $R$-symmetry U(1) gauge field, and thus the fermions remain charged. The resulting theory is shown in fact to be equivalent to the Freedman model on-shell. ${ }^{1}$

The outline of the paper is the following. In section 2 , we discuss the $D$-deformation in $\mathcal{N}=1$ global supersymmetry and show that it corresponds to a magnetic Fayet-Iliopoulos term. In section 3, we shortly review the new-minimal formulation of pure supergravity [7, $9,10]$ needed in the following. In section 4 , we construct the coupling of the deformed super-Maxwell theory to supergravity using the method described above which consists of modifying the Bianchi identity of a chiral spinor superfield by a term proportional to the compensator linear multiplet with a constant coefficient playing the role of the integration constant in global supersymmetry. In section 5, we describe the introduction of the FayetIliopoulos term in new-minimal supergravity and show how this formulation is related to an old-minimal theory with the Maxwell multiplet used to gauge the $\mathrm{U}(1)_{R}$ superconformal symmetry, following for instance ref. [11]. This is the Freedman model [12]. We then compare it with the deformed super-Maxwell theory and verify that the two theories are indentical once auxiliary fields have been eliminated. In section 6 , we work out the EM duality in supergravity, generalising the result of global supersymmetry and we show that in the absence of matter the deformed super-Maxwell theory is dual to the Freedman model of gauged $R$-symmetry. Section 7 contains a summary of our results and an outlook. The paper is also accompanied by three appendices: appendix A contains our conventions and useful formulae; appendix B describes the local superconformal chiral spinor multiplet used in the main body of the paper; appendix $\mathrm{C}$ is devoted to a complementary description of the deformed Maxwell theory in curved superspace.

\section{$2 \quad D$-deformation in global $\mathcal{N}=1$ supersymmetry}

The $\mathcal{N}=1$ super-Maxwell theory is usually formulated in terms of a chiral spinor superfield $W_{\alpha}$ subject to the superfield condition ${ }^{2}$

$$
D^{\alpha} W_{\alpha}=\bar{D}_{\dot{\alpha}} \bar{W}^{\dot{\alpha}}
$$

which imposes the Bianchi identity $\partial_{[\mu} F_{\nu \rho]}=0$ on the Maxwell field-strength $F_{\mu \nu}$.

Consider instead an arbitrary chiral spinor superfield $\Upsilon_{\alpha}, \bar{D}_{\dot{\alpha}} \Upsilon_{\beta}=0$ and its conjugate $\bar{\Upsilon}_{\dot{\alpha}}=\left(\Upsilon_{\alpha}\right)^{*}$. The superfield $D^{\alpha} \Upsilon_{\alpha}-\bar{D}_{\dot{\alpha}} \bar{\Upsilon}^{\dot{\alpha}}$ is real and linear $[16,17]$. The condition

$$
D^{\alpha} \Upsilon_{\alpha}-\bar{D}_{\dot{\alpha}} \bar{\Upsilon}^{\dot{\alpha}}=L
$$

\footnotetext{
${ }^{1}$ Using BRST methods to study deformations of $\mathcal{N}=1$ supergravity, a similar but not identical model has been presented in $[14,15]$.

${ }^{2}$ We use covariant derivatives

$$
D_{\alpha}=\frac{\partial}{\partial \theta^{\alpha}}-i\left(\sigma^{\mu} \bar{\theta}\right)_{\alpha} \partial_{\mu}, \quad \bar{D}_{\dot{\alpha}}=\frac{\partial}{\partial \bar{\theta}^{\dot{\alpha}}}-i\left(\theta \sigma^{\mu}\right)_{\dot{\alpha}} \partial_{\mu}
$$
}


defines $\Upsilon^{\alpha}$ for a given $L, \overline{D D} L=0$, up to a solution $\Upsilon_{0 \alpha}$ of eq. (2.1), which is ${ }^{3}$

$$
\Upsilon_{0 \alpha}=i W_{\alpha}, \quad \bar{\Upsilon}_{0 \dot{\alpha}}=i \bar{W}_{\dot{\alpha}}, \quad W_{\alpha}=-\frac{1}{4} \overline{D D} D_{\alpha} V, \quad \bar{W}_{\dot{\alpha}}=-\frac{1}{4} D D \bar{D}_{\dot{\alpha}} V,
$$

where $V$ is a real superfield. In the simplest case, we can take $L$ to be a (real) constant, $L=4 \zeta$. This amounts to give a supersymmetric-invariant background value to the lowest scalar component of $L$. The solution of

$$
D^{\alpha} \Upsilon_{\alpha}-\bar{D}_{\dot{\alpha}} \bar{\Upsilon}^{\dot{\alpha}}=4 \zeta
$$

is

$$
\Upsilon_{\alpha}=-\zeta \theta_{\alpha}+i W_{\alpha} \equiv i W_{\alpha}^{\text {def }}, \quad \bar{\Upsilon}_{\dot{\alpha}}=-\zeta \bar{\theta}_{\dot{\alpha}}+i \bar{W}_{\dot{\alpha}} \equiv i \bar{W}_{\dot{\alpha}}^{\mathrm{def}}
$$

where the deformed chiral Maxwell superfield is

$$
W_{\alpha}^{\text {def }}=-i \lambda_{\alpha}+\theta_{\alpha}(D+i \zeta)-\frac{i}{2}\left(\sigma^{\mu} \bar{\sigma}^{\nu} \theta\right)_{\alpha} F_{\mu \nu}-\theta \theta\left(\sigma^{\mu} \partial_{\mu} \bar{\lambda}\right)_{\alpha}
$$

and satisfies the deformed supersymmetric Bianchi identity $D^{\alpha} W_{\alpha}^{\text {def }}-\bar{D}_{\dot{\alpha}} \bar{W}^{\text {def } \dot{\alpha}}=-4 i \zeta$. The supersymmetry variation of the gaugino is now

$$
\delta \lambda_{\alpha}=-\zeta \epsilon_{\alpha}+i D \epsilon_{\alpha}-\frac{1}{4} \epsilon\left[\sigma^{\mu}, \bar{\sigma}^{\nu}\right] F_{\mu \nu} .
$$

Therefore $\lambda$ appears to transform like a Goldstino because of the deformation.

To see the role of the deformation, consider the lagrangian

$$
\mathcal{L}=-\frac{1}{2} \operatorname{Im}\left[\widetilde{\tau} \int d^{2} \theta \Upsilon^{2}\right]-\frac{1}{2} \int d^{2} \theta d^{2} \bar{\theta} U\left(D^{\alpha} \Upsilon_{\alpha}-\bar{D}_{\dot{\alpha}} \bar{\Upsilon}^{\dot{\alpha}}-4 \zeta\right), \quad \widetilde{\tau}=\frac{i}{\tilde{g}^{2}}+\vartheta,
$$

where $\Upsilon_{\alpha}$ is a chiral spinor superfield without extra constraint and $U$ is a real scalar superfield.

Eliminating $U$ imposes the constraint (2.4) on $\Upsilon_{\alpha}$ and leads to a deformed Maxwell theory

$$
\mathcal{L}_{M}=\frac{1}{2} \operatorname{Im}\left[\widetilde{\tau} \int d^{2} \theta W_{\mathrm{def}}^{2}\right]=\frac{1}{2} \operatorname{Im}\left[\widetilde{\tau} \int d^{2} \theta W^{2}\right]+\frac{1}{2}\left(-\zeta^{2} \operatorname{Im} \widetilde{\tau}+2 \zeta D \operatorname{Re} \widetilde{\tau}\right),
$$

where we used $W_{\alpha}^{\text {def }}=W_{\alpha}+i \zeta \theta_{\alpha}$. Notice the emergence of a Fayet-Iliopoulos term proportional to the theta-angle $\operatorname{Re} \widetilde{\tau}$, induced by the deformation, as was noticed in the context of $\mathcal{N}=2[3,4]$.

Alternatively, we can integrate by parts and rewrite the lagrangian (2.8) as

$$
\mathcal{L}=-\frac{1}{2} \operatorname{Im} \int d^{2} \theta\left(\widetilde{\tau} \Upsilon^{2}+\frac{i}{2} \Upsilon^{\alpha} \overline{D D} D_{\alpha} U\right)+2 \zeta \int d^{2} \theta d^{2} \bar{\theta} U
$$

Eliminating $\Upsilon$, we arrive at

$$
\mathcal{L}_{E}=\frac{1}{2} \operatorname{Im}\left[\tau \int d^{2} \theta W^{2}\right]+2 \zeta \int d^{2} \theta d^{2} \bar{\theta} U, \quad W_{\alpha}=-\frac{1}{4} \overline{D D} D_{\alpha} U ; \quad \tau=-\frac{1}{\widetilde{\tau}} .
$$

\footnotetext{
${ }^{3}$ With this convention, $\bar{W}_{\dot{\alpha}}=-\left(W_{\alpha}\right)^{*}$.
} 
The last lagrangian (2.11) is the standard "electric" expression of the super-Maxwell theory with a Fayet-Iliopoulos term. It is the electric dual of the "magnetic" lagrangian (2.9), with the deformation induced by $\zeta$. In both the electric and magnetic descriptions, a constant value

$$
-\frac{\zeta^{2}}{2} \frac{\widetilde{\tau} \bar{\tau}}{\operatorname{Im} \widetilde{\tau}}=-\frac{\zeta^{2}}{2} \frac{1}{\operatorname{Im} \tau}
$$

is added to the super-Maxwell lagrangian, after eliminating $D$. The Fayet-Iliopoulos term can be seen as a nonlinear deformation of $\delta \lambda$ induced by a constant $\langle D\rangle$. In the magnetic dual, the deformation is induced by the parameter $\zeta$ introduced in the Bianchi identity (2.4). As expected, the electric and magnetic couplings are related by $\tau \leftrightarrow-1 / \tau \equiv \widetilde{\tau}$. At the superfield level, the magnetic (2.9) and the electric (2.11) versions are related by the map of field strengths $W_{\alpha} \longleftrightarrow \widetilde{\tau} W_{\alpha}^{\text {def }}{ }^{4}$

To summarise, the above argument shows that the deformation in the Bianchi identity is equivalent to a Fayet-Iliopoulos term in the 'magnetic' dual theory. The constant term (2.12), which is irrelevant in the context of global supersymmetry, acquires relevance when the theory is coupled to $\mathcal{N}=1$ supergravity or if $\tau$ is promoted to an analytic function of some neutral chiral superfields. It is then interesting to evaluate the scalar potential obtained in the presence of both 'electric' and 'magnetic' Fayet-Iliopoulos terms. This is possible by adding in the deformed theory (2.9) a term linear in the auxiliary field $D$ with a constant coefficient $\xi$, which transforms with a total derivative under supersymmetry. In this case, the constant (2.12) is replaced by the scalar potential

$$
V=\frac{1}{2}\left\{\frac{(\xi+\zeta \operatorname{Re} \widetilde{\tau})^{2}}{\operatorname{Im} \widetilde{\tau}}+\zeta^{2} \operatorname{Im} \widetilde{\tau}\right\}
$$

which is invariant under $\tau \leftrightarrow-1 / \tau$ (or $\widetilde{\tau} \leftrightarrow-1 / \widetilde{\tau}$ ) and the exchange $(\xi, \zeta) \rightarrow(\zeta,-\xi)$. This contribution can be obtained from an $\mathcal{N}=2$ supersymmetric theory by restricting the electric and magnetic triplets of Fayet-Iliopoulos terms to the $D$-direction and identify $\tau$ with the second derivative of the prepotential $[3,4]$. Note that it has a runaway behaviour towards strong coupling $(\operatorname{Im} \tilde{\tau}=0)$ after minimisation with respect to the theta-angle $\vartheta=\operatorname{Re} \tilde{\tau}$, when minimising with respect to $\tilde{g}$. A non-trivial superpotential is needed to stabilise the theory.

Another question concerns the addition of charged matter. This is straightforward in the dual version of the theory containing a Fayet-Iliopoulos term. On the contrary, it is not possible to add charged matter in the presence of the deformation. One can infer this from the fact that the real vector superfield needed to describe gauge-invariant kinetic matter lagrangians cannot include the deformation. ${ }^{5}$ Consider the standard real and gauge invariant $\bar{\Phi} e^{V} \Phi$ used to couple a chiral multiplet (with U(1) charge one) to the real Maxwell superfield $V$. The kinetic lagrangian includes a Yukawa interaction involving the gaugino:

$$
\bar{\Phi} e^{V} \Phi \quad \longrightarrow \quad \frac{i}{\sqrt{2}}(\bar{z} \lambda \psi-z \overline{\lambda \psi}) .
$$

\footnotetext{
${ }^{4}$ See refs. [5, 18] for more detail about electric-magnetic duality transformations in superspace with and without Fayet-Iliopoulos term and Bianchi identity deformations.

${ }^{5}$ In other words, $W_{\alpha}^{\text {def }}$ in eq. (2.5) cannot be written as $-\frac{1}{4} \overline{D D} D_{\alpha} V$, with a real $V$.
} 
Its deformed variation is

$$
\frac{i}{\sqrt{2}}\left(\bar{z}\left(\delta_{\mathrm{def}} \lambda\right) \psi-z\left(\delta_{\mathrm{def}} \bar{\lambda}\right) \bar{\psi}\right)=-\frac{i}{\sqrt{2}} \zeta(\bar{z} \epsilon \psi-z \overline{\epsilon \psi})=-i \zeta(\bar{z} \delta z-z \delta \bar{z})
$$

where $\delta_{\text {def }} \lambda_{\alpha}=-\zeta \epsilon_{\alpha}, \delta_{\text {def }} \bar{\lambda}_{\dot{\alpha}}=-\zeta \bar{\epsilon}_{\dot{\alpha}}$. This variation cannot be cancelled by a $\zeta$-dependent counterterm added to the lagrangian. This simple argument easily generalizes to an arbitrary Kähler potential.

It follows that in the case of several U(1)'s with different deformation parameters $\zeta_{i}$, charged matter fields should satisfy the requirement of being neutral under the U(1) combination containing the deformation, while non trivial charges can exist only with respect to the remaining orthogonal combinations for which supersymmetry variations are not deformed. This requirement is translated to the follwing condition on the physical charges $q_{i}$ :

$$
\sum_{i} q_{i} \zeta_{i}=0
$$

where the various gauge couplings are implicit in the definition of the charges.

This apparent obstruction seems to have an important physical implication for magnetic monopoles. Indeed, states with magnetic charges may be in principle added in the theory with a deformation in the electric Bianchi identity (although its local description is not known), since they correspond to electrically charged states in the dual theory with a Fayet-Iliopoulos term. On the other hand, magnetically charged states seem to be forbidden for a U(1) with an electric Fayet-Iliopoulos term, since they would correspond to electrically charged matter in the deformed theory in view of the obstruction described above.

In the context of global supersymmetry without coupling to matter, which is the focus of our paper, the above discussion is not fruitful: we are merely considering a free theory. It acquires relevance when coupled to $\mathcal{N}=1$ supergravity that we study in the following.

\section{Supergravity}

In the previous section, we have introduced in global supersymmetry a deformation of super-Maxwell theory which is the magnetic dual of the standard, electric, Fayet-Iliopoulos term. In the rest of the paper, we extend this construction to supergravity. We use a superconformal formulation, ${ }^{6}$ which is certainly appropriate to describe the super-Maxwell system, and, since the idea is to use the linear multiplet $L$ of eq. (2.2) as compensating multiplet, we use the new-minimal formulation of $\mathcal{N}=1$ supergravity $[9,10] .{ }^{7}$ As usual, the resulting new-minimal theory can be transformed (before Poincaré gauge fixing) into the old-minimal one by a superconformal chiral-linear duality transformation [11].

The superconformal formulation of new-minimal $\mathcal{N}=1$ supergravity uses a real linear multiplet $L$ as compensator $[7,8,11]$. Its lowest component $C$ is used to gauge-fix Weyl

\footnotetext{
${ }^{6}$ Reviewed in ref. [13].

${ }^{7}$ By definition, minimal supergravity has an off-shell multiplet of fields with the gravitino as the sole fermion. It has then 12 bosonic and 12 fermionic component fields $\left(12_{B}+12_{F}\right)$. There are two choices of auxiliary fields, leading to old- [19-22] and new-minimal [9, 10] supergravity.
} 
symmetry with the condition $C=\kappa^{-2}$. Hence, one can transport the discussion of global supersymmetry to new-minimal supergravity by defining a chiral spinor multiplet with weights $w=n=3 / 2^{8}$ through the equation ${ }^{9}$

$$
D^{\alpha} \Upsilon_{\alpha}-\bar{D}_{\dot{\alpha}} \bar{\Upsilon}^{\dot{\alpha}}=4 \zeta L .
$$

As a consequence, $\Upsilon_{\alpha}$ includes the Maxwell multiplet and the "prepotential" of the compensating real linear multiplet $L$. Gauge-fixing superconformal symmetry will then generate a deformation parameter $4 \zeta \kappa^{-2}$ in the Poincaré theory, completely analogous to $4 \zeta$ in the global case.

\subsection{New minimal supergravity}

The auxiliary fields of the $12_{B}+12_{F}$ off-shell multiplet of new-minimal supergravity are $[9$, 10] an antisymmetric tensor $B_{\mu \nu}$ and a vector field $A_{\mu}$. Both are gauge fields and contain $3_{B}+3_{B}$ field components. The vector $A_{\mu}$ is the superconformal $\mathrm{U}(1)_{R}$ gauge field, and $B_{\mu \nu}$ is in the linear compensating multiplet $L$ together with the real scalar $C$ and Majorana fermion $\chi$. It is convenient to describe the linear multiplet $L$ as a real multiplet with weight $w=2, n=0$ and components:

$$
L=\left(C, \quad \chi, \quad 0, \quad-E_{a}, \quad-\gamma^{b} D_{b} \chi, \quad-\square C\right), \quad D^{a} E_{a}=0 .
$$

The constraint $D^{a} E_{a}=0$ can be solved explicitly in terms of $B_{a b}$ and the solution is given in eq. (B.10). The superconformal construction [7] of the new-minimal theory $[9,10]$ is based on the lagrangian

$$
\mathcal{L}_{\text {new-min }}=\frac{3}{2}\left[L \ln \frac{L}{\bar{S} S}-L\right]_{D},
$$

where $S$ is a non-dynamical chiral multiplet with $w=n=1$ and $[\ldots]_{D}$ is the real invariant density formula for a real multiplet with $w=2 .{ }^{10}$ Since $[L(\Lambda+\bar{\Lambda})]_{D}$ is a derivative for a chiral multiplet $\Lambda$ with $w=n=0$, the action is invariant under the gauge transformation

$$
S \quad \longrightarrow \quad e^{\Lambda} S
$$

even if this gauge symmetry is not explicitly gauged. ${ }^{11}$ Since the zero-weight real multiplet $\ln \frac{L}{\overline{S S}}$ transforms under (3.4) as a Maxwell vector multiplet, one can take the Wess-Zumino gauge by an appropriate choice of the components $\varphi, \psi_{L}$ and $f$ of the chiral multiplet $S$. In terms of the components $C, \chi$ and $B_{\mu \nu}$ of $L$, the choice is

$$
\varphi=\sqrt{C} e^{i \alpha}, \quad \psi_{L}=\frac{i}{\sqrt{2 C}} e^{i \alpha} \chi_{L}, \quad f=0
$$

\footnotetext{
${ }^{8}$ We denote by $w$ and $n$ the Weyl weight and the $\mathrm{U}(1)_{R}$ charge of a field or a multiplet. The normalization of $\mathrm{U}(1)_{R}$ is such that $w=n$ for (the lowest component of) a chiral multiplet. Note that, we mostly follow the conventions of ref. [13], with some exceptions stated in appendix A.

${ }^{9} \mathrm{By} D^{\alpha} \Upsilon_{\alpha}$ or $\bar{D}_{\dot{\alpha}} \bar{\Upsilon}^{\dot{\alpha}}$, we mean the local superconformal multiplets with weights $w=2, n=0$ corresponding to the global superfields $D^{\alpha} \Upsilon_{\alpha}$ or $\bar{D}_{\dot{\alpha}} \bar{\Upsilon}^{\dot{\alpha}}$. A curved superspace meaning to the covariant derivatives $D^{\alpha}$ and $\bar{D}_{\dot{\alpha}}$ can be given by using a "conformal superspace" approach [23] to $\mathcal{N}=1$ conformal supergravity, see appendix C.

${ }^{10}$ The term linear in $L$ is added for convenience: it only contributes with a derivative.

${ }^{11}$ An explicit gauging for the chiral multiplet is given by $\bar{S} e^{V} S$ with $V$ being the gauge multiplet.
} 
which as usual leaves arbitrary the phase $\alpha$ of $\varphi$ which always appears in the combination $A_{\mu}-\partial_{\mu} \alpha$. It thus can be eliminated with a $\mathrm{U}(1)_{R}$ gauge transformation. Note that this procedure respects the superconformal $\mathrm{U}(1)_{R}$ symmetry. Poincaré gauge fixing applied to the components $C, \chi$ and $B_{\mu \nu}$ of $L$ is then

$$
C=\frac{1}{\kappa^{2}} \quad(\text { Weyl }), \quad \chi=0 \quad(\mathcal{S} \text { supersymmetry }) .
$$

In addition, conformal boosts are fixed by the choice $b_{\mu}=0$ (Weyl gauge field). With these choices and gauge fixings for $S$ and $L$, one can easily use the $D$-density formula (A.5) to compute the component expansion of the lagrangian (3.3) and obtain the pure new-minimal Poincaré supergravity off-shell theory. These gauge-fixing conditions will be used in the rest of this section.

There are two useful expressions of the new-minimal Poincaré theory. The first is

$$
\mathcal{L}_{\text {new-min }}=\frac{e}{2 \kappa^{2}}\left[R-\bar{\psi}_{\mu} \gamma^{\mu \nu \rho} \mathcal{D}_{\nu} \psi_{\rho}\right]-\frac{3 \kappa^{2}}{4} e E^{a} E_{a}-\frac{3}{2} e A_{\mu} \epsilon^{\mu \nu \rho \sigma} \partial_{\nu} B_{\rho \sigma}
$$

where $R=e_{a}^{\mu} e_{b}^{\nu} R_{\mu \nu}^{a b}(\omega(e, \psi))$,

$$
E^{\mu}=\frac{1}{2} \epsilon^{\mu \nu \rho \sigma} \partial_{\nu} B_{\rho \sigma}+\frac{1}{4 \kappa^{2}} \epsilon^{\mu \nu \rho \sigma} \bar{\psi}_{\rho} \gamma_{\sigma} \psi_{\nu}
$$

and

$$
\mathcal{D}_{\nu} \psi_{\rho}=\mathcal{D}_{\nu}^{(P)} \psi_{\rho}-\frac{3}{2} i A_{\nu} \gamma_{5} \psi_{\rho}, \quad \mathcal{D}_{\nu}^{(P)} \psi_{\rho}=\partial_{\nu} \psi_{\rho}+\frac{1}{8} \omega_{\nu a b}(e, \psi)\left[\gamma^{a}, \gamma^{b}\right] \psi_{\rho} .
$$

Note for future use that eq. (3.8) solves the constraint

$$
\partial_{\mu}\left(e E^{\mu}-\frac{i e}{4 \kappa^{2}} \bar{\psi}_{\nu} \gamma^{\mu \nu \rho} \gamma_{5} \psi_{\rho}\right)=0
$$

where the second term includes the divergence of the gravitino chiral current. This first form (3.7) of the new-minimal action is explicitly invariant under $\mathrm{U}(1)_{R}$, with lagrangian variation

$$
\delta_{R} \mathcal{L}_{\text {new min }}=-\frac{3}{2} \partial_{\mu}\left[e \lambda_{T} \epsilon^{\mu \nu \rho \sigma} \partial_{\nu} B_{\rho \sigma}\right]
$$

induced by the $A_{\mu}$ term is eq. (3.7).

The second equivalent expression useful to eliminate auxiliary fields is

$$
\begin{aligned}
\mathcal{L}_{\text {new-min }} & =\frac{e}{2 \kappa^{2}}\left[R-\bar{\psi}_{\mu} \gamma^{\mu \nu \rho} \mathcal{D}_{\nu}^{(P)} \psi_{\rho}\right]-\frac{3 \kappa^{2}}{4} e E^{a} E_{a}-3 e A_{a} E^{a} \\
& =\frac{e}{2 \kappa^{2}}\left[R-\bar{\psi}_{\mu} \gamma^{\mu \nu \rho} \mathcal{D}_{\nu}^{(P)} \psi_{\rho}\right]-3 e A_{a}^{\prime} E^{a},
\end{aligned}
$$

defining a shifted $\mathrm{U}(1)_{R}$ gauge field as

$$
A_{a}^{\prime}=A_{a}+\frac{\kappa^{2}}{4} E_{a}
$$

In pure supergravity, the equations of motion of the auxiliary fields lead to $E_{a}=0$ and $A_{a}=A_{a}^{\prime}=$ pure gauge, which then also vanishes by a gauge choice. 
The spin connection $\omega_{\mu}^{a b}=\omega_{\mu}^{a b}(e, \psi)$ solves the constraint $R_{\mu \nu}^{a}(P)=0$ on the curvature of space-time translations, with $b_{\mu}=0$. It decomposes into

$$
\omega_{\mu}^{a b}=\omega_{\mu}^{a b}(e, \psi)=\omega_{\mu}^{a b}(e)+\kappa_{\mu}^{a b},
$$

with Poincaré spin connection

$$
\omega_{\mu}^{a b}(e)=-\frac{1}{2}\left(\partial_{\mu} e_{\nu}^{a}-\partial_{\nu} e_{\mu}^{a}\right) e^{\nu b}+\frac{1}{2}\left(\partial_{\mu} e_{\nu}^{b}-\partial_{\nu} e_{\mu}^{b}\right) e^{\nu a}-\frac{1}{2} e^{\rho a} e^{\sigma b}\left(\partial_{\rho} e_{\sigma}^{c}-\partial_{\sigma} e_{\rho}^{c}\right) e_{\mu c}
$$

and contorsion tensor

$$
\kappa_{\mu}^{a b}=\frac{1}{4}\left[\bar{\psi}_{\mu} \gamma^{a} \psi^{b}+\bar{\psi}^{a} \gamma_{\mu} \psi^{b}-\bar{\psi}_{\mu} \gamma^{b} \psi^{a}\right]
$$

When the theory is expressed in terms of the Poincaré spin connection $\omega_{\mu}^{a b}(e)$, the kinetic supergravity lagrangian produces the usual four-gravitino interactions

$$
\mathcal{L}_{4, \mathrm{SG}}=\frac{e}{16 \kappa^{2}}\left[4\left(\bar{\psi}_{\mu} \gamma^{\mu} \psi_{\rho}\right)\left(\bar{\psi}_{\nu} \gamma^{\nu} \psi^{\rho}\right)-\left(\bar{\psi}_{\mu} \gamma_{\nu} \psi_{\rho}\right)\left(\bar{\psi}^{\mu} \gamma^{\nu} \psi^{\rho}\right)-2\left(\bar{\psi}_{\mu} \gamma_{\nu} \psi_{\rho}\right)\left(\bar{\psi}^{\mu} \gamma^{\rho} \psi^{\nu}\right)\right]
$$

\section{The deformed super-Maxwell theory in supergravity}

The description of the deformed Maxwell theory in conformal supergravity uses a full chiral spinor multiplet $\Upsilon$ with weight $w=n=3 / 2$ and with $8_{B}+8_{F}$ off-shell field components. As seen in eq. (3.1) which has a superconformal version and in appendix B, it combines the super-Maxwell fields and the linear multiplet $L$. Then, as outlined in the global case, applying the Poincaré gauge-fixing conditions (3.6) to $L$ used as compensating multiplet will generate a deformation in the Poincaré supergravity theory.

The field content of the chiral spinor superfield includes the real scalars $C$ and $D$, the two-form (non-gauge) field $\mathcal{B}_{a b}$ and two Majorana spinors $\lambda$ (gaugino) and $\chi$. Since this superconformal multiplet does not seem available in the literature, its supersymmetry variations are given in appendix B. It includes two submultiplets: the Maxwell multiplet with fields $\lambda, D, \mathcal{A}_{a}$ (with field-strength $\mathcal{F}_{a b}$ ), and the linear multiplet with fields $C, \chi$ and the gauge field $B_{\mu \nu}$. Its decomposition into these two $4_{B}+4_{F}$ submultiplets is consistent. In particular, the vector field $E^{a}$ present in the supersymmetry variations is defined as $E^{a}=\frac{1}{2} \epsilon^{a b c d} \mathcal{D}_{b} \mathcal{B}_{c d}$ in the chiral spinor multiplet and as the solution of $\mathcal{D}^{a} E_{a}=0$ in the linear multiplet, with the covariant derivatives relevant to each multiplet. That both definitions lead to the same expression depending on $B_{\mu \nu}, C$ and $\chi$ follows from the Bianchi identity holding among components of the Maxwell submultiplet. This argument also implies the decomposition $\mathcal{B}_{\mu \nu}=B_{\mu \nu}-\widehat{\mathcal{F}}_{\mu \nu}$ with the superconformal Maxwell field-strength

$$
\widehat{\mathcal{F}}_{\mu \nu}=\partial_{\mu} \mathcal{A}_{\nu}-\partial_{\nu} \mathcal{A}_{\mu}+\frac{1}{2} \bar{\psi}_{\mu} \gamma_{\nu} \lambda-\frac{1}{2} \bar{\psi}_{\nu} \gamma_{\mu} \lambda
$$

where we use the symbol hat to denote the superconformal field strength, including fermions. 
To characterize the interaction of the linear submultiplet identified as the compensator of new-minimal supergravity with the super-Maxwell fields, we need a coupling constant $\zeta$. It is simply introduced by rescaling the components of the linear multiplet

$$
C, \quad \chi, \quad B_{\mu \nu} \quad \longrightarrow \quad \zeta C, \quad \zeta \chi, \quad \zeta B_{\mu \nu}, \quad \mathcal{B}_{\mu \nu}=\zeta B_{\mu \nu}-\widehat{\mathcal{F}}_{\mu \nu},
$$

within the chiral spinor multiplet. The parameter $\zeta$ will characterize the supersymmetry deformation in the final lagrangian. Note for future use that the vector field (3.8) becomes

$$
E^{\mu}=\frac{1}{2 \zeta} \epsilon^{\mu \nu \rho \sigma} \partial_{\nu} \mathcal{B}_{\rho \sigma}+\frac{1}{2 \zeta} \epsilon^{\mu \nu \rho \sigma} \partial_{\nu}\left(\bar{\psi}_{\rho} \gamma_{\sigma} \lambda\right)+\frac{1}{4 \kappa^{2}} \epsilon^{\mu \nu \rho \sigma} \bar{\psi}_{\rho} \gamma_{\sigma} \psi_{\nu}
$$

Since the square of the chiral spinor multiplet $\Upsilon^{2}$ is chiral with weight $w=3$, its components can be introduced in the superconformal $F$-density action formula, $\left[\Upsilon^{2}\right]_{F}$. The deformed super-Maxwell theory is defined as

$$
\mathcal{L}_{\text {def Maxwell }}=-\frac{1}{2} \operatorname{Re}\left[\Upsilon^{2}\right]_{F}-\frac{\vartheta}{2} \operatorname{Im}\left[\Upsilon^{2}\right]_{F},
$$

whose explicit expression is computed in (B.23). The real part provides the super-Maxwell lagrangian with canonically-normalized kinetic terms and the imaginary part introduces an arbitrary parameter $\vartheta$. The theory has then two parameters, $\zeta$ and $\vartheta$. Adding a coupling constant factor $g^{-2}$ to the first term is not necessary since it can be absorbed in $\Upsilon$ and is not observable. ${ }^{12}$ After some work to rearrange four-fermion terms and applying the Poincaré gauge-fixing conditions (3.6), one finds

$$
\begin{aligned}
-\frac{1}{2} e^{-1} \operatorname{Re}\left[\Upsilon^{2}\right]_{F}= & -\frac{1}{4} \mathcal{B}_{a b} \mathcal{B}^{a b}-\frac{1}{2} \bar{\lambda} \gamma^{a} D_{a} \lambda+\frac{1}{2} D^{2}-\frac{\zeta^{2}}{2 \kappa^{2}} \\
& +\frac{1}{4} \bar{\psi}_{\mu} \gamma^{\mu}\left(\frac{\zeta}{\kappa^{2}}-i D \gamma_{5}\right) \lambda-\frac{1}{16} \mathcal{B}_{a b} \bar{\psi}_{\mu} \gamma^{\mu}\left[\gamma^{a}, \gamma^{b}\right] \lambda \\
& -\frac{1}{8}\left(\bar{\psi}_{\mu} \gamma_{\nu} \lambda\right)\left(\bar{\psi}_{\rho} \gamma^{\rho \mu \nu} \lambda\right) .
\end{aligned}
$$

At this stage,

$$
D_{\mu} \lambda=\partial_{\mu} \lambda-\frac{3}{2} i A_{\mu} \gamma_{5} \lambda+\frac{1}{8} \omega_{\mu a b}\left[\gamma^{a}, \gamma^{b}\right] \lambda+\frac{1}{2 \kappa^{2}} \psi_{\mu}-\frac{i}{2} D \gamma_{5} \psi_{\mu}+\frac{1}{8}\left[\gamma^{a}, \gamma^{b}\right] \psi_{\mu} \mathcal{B}_{a b}
$$

Defining the Poincaré-covariant derivative

$$
\mathcal{D}_{\mu}^{(P)} \lambda=\partial_{\mu} \lambda+\frac{1}{8} \omega_{\mu a b}\left[\gamma^{a}, \gamma^{b}\right] \lambda
$$

with spin connection $\omega_{\mu a b}$ given by eq. (3.14) leads to the deformed super-Maxwell theory

$$
\begin{aligned}
-\frac{1}{2} e^{-1} \operatorname{Re}\left[\Upsilon^{2}\right]_{F}= & -\frac{1}{4} \mathcal{B}_{a b} \mathcal{B}^{a b}-\frac{1}{2} \bar{\lambda} \gamma^{a} \mathcal{D}_{a}^{(P)} \lambda+\frac{1}{2} D^{2}+\frac{3}{4} i \bar{\lambda} \gamma^{a} \gamma_{5} \lambda A_{a}-\frac{\zeta^{2}}{2 \kappa^{4}} \\
& -\frac{1}{4} \bar{\psi}_{c} \gamma^{c a b} \lambda \mathcal{B}_{a b}+\frac{\zeta}{2 \kappa^{2}} \bar{\psi}_{\mu} \gamma^{\mu} \lambda-\frac{1}{8}\left(\bar{\psi}_{\mu} \gamma_{\nu} \lambda\right)\left(\bar{\psi}_{\rho} \gamma^{\rho \mu \nu} \lambda\right) .
\end{aligned}
$$

\footnotetext{
${ }^{12}$ We will return to this at the end of the section.
} 
For $\zeta=0$, the lagrangian (4.3) reduces to the one of super-Maxwell theory, with also $\mathcal{B}_{a b}=-\widehat{\mathcal{F}}_{a b}$. But for $\zeta \neq 0$ the Maxwell gauge field $\mathcal{A}_{\mu}$ is not expected to propagate degrees of freedom since it can be eliminated by a gauge variation of $B_{\mu \nu}$.

If the Poincaré spin connection $\omega_{\mu a b}(e)$ is used in expression (4.7), a second four-fermion terms is generated by the contorsion tensor (3.16) located in the Dirac kinetic lagrangian. Using $\bar{\lambda} \gamma^{a} \lambda=0$, it reads

$$
-\frac{1}{8} \kappa_{a b c} \bar{\lambda} \gamma^{a b c} \lambda=-\frac{1}{32}\left(\bar{\lambda} \gamma^{a b c} \lambda\right)\left(\bar{\psi}_{a} \gamma_{b} \psi_{c}\right)
$$

The fact that supersymmetry is broken when $C=\kappa^{-2}$ follows from the presence of a constant, nonlinear term in the gaugino variation:

$$
\delta \lambda=-\frac{1}{2}\left(\frac{\zeta}{\kappa^{2}}-i D \gamma_{5}\right) \epsilon+\text { linear }
$$

which also shows again that the $\zeta$-deformation cannot be absorbed or generated by a Fayet-Iliopoulos term inducing $\langle D\rangle \neq 0$. In addition, since

$$
\delta \chi=-\frac{2 i}{\kappa^{2}} \gamma_{5} \eta+\frac{1}{2} E_{a} \gamma^{a} \epsilon,
$$

the invariance of the Poincaré fixing condition $\chi=0$ implies that the Poincaré supersymmetry has a parameter $\epsilon_{P}$ which combines $\epsilon \equiv \epsilon_{P}$ ( $\mathcal{Q}$ supersymmetry) with a $\mathcal{S}$ supersymmetry variation with parameter $\eta=\frac{i \kappa^{2}}{4} E^{a} \gamma_{a} \gamma_{5} \epsilon_{P}$.

The $\vartheta$-term in the lagrangian (4.3) is

$$
\begin{aligned}
-\frac{\vartheta}{2} \operatorname{Im} \Upsilon^{2}=\vartheta & {\left[-\frac{e}{8} \epsilon^{\mu \nu \rho \sigma}\left(\mathcal{B}_{\mu \nu}+\bar{\psi}_{\mu} \gamma_{\nu} \lambda\right)\left(\mathcal{B}_{\rho \sigma}+\bar{\psi}_{\rho} \gamma_{\sigma} \lambda\right)+\frac{e}{\kappa^{2}} \zeta D\right.} \\
& \left.-\frac{i e}{2 \kappa^{2}} \zeta \bar{\psi}_{\mu} \gamma^{\mu} \gamma_{5} \lambda-\frac{i}{4} \partial_{\mu}\left(e \bar{\lambda} \gamma^{\mu} \gamma_{5} \lambda\right)\right] .
\end{aligned}
$$

It depends on

$$
\mathcal{B}_{\mu \nu}+\frac{1}{2} \bar{\psi}_{\mu} \gamma_{\nu} \lambda-\frac{1}{2} \bar{\psi}_{\nu} \gamma_{\mu} \lambda=\zeta B_{\mu \nu}-\partial_{\mu} \mathcal{A}_{\nu}+\partial_{\nu} \mathcal{A}_{\mu}
$$

and for $\zeta=0$ it reduces to the super-Maxwell expression

$$
\begin{aligned}
-\frac{\vartheta}{2} \operatorname{Im} \Upsilon_{\text {Maxwell }}^{2} & =-\frac{e}{8} \vartheta \epsilon^{\mu \nu \rho \sigma} \mathcal{F}_{\mu \nu} \mathcal{F}_{\rho \sigma}-\frac{i}{4} \vartheta \partial_{\mu}\left(e \bar{\lambda} \gamma^{\mu} \gamma_{5} \lambda\right) \quad ; \quad \mathcal{F}_{\mu \nu}=\partial_{\mu} \mathcal{A}_{\nu}-\partial_{\nu} \mathcal{A}_{\mu} \\
& =-\frac{\vartheta}{2} \partial_{\mu}\left(e \epsilon^{\mu \nu \rho \sigma} \mathcal{A}_{\nu} \partial_{\rho} \mathcal{A}_{\sigma}+\frac{i}{2} e \bar{\lambda} \gamma^{\mu} \gamma_{5} \lambda\right)
\end{aligned}
$$

which is a total derivative. If the theory is deformed by coupling the Maxwell theory to the compensator, i.e. if $\zeta \neq 0$, it also naturally includes a Fayet-Iliopoulos term $e \kappa^{-2} \vartheta \zeta D$ with free parameter $\vartheta$. Since it is a derivative for $\zeta=0$, the $\vartheta$-term generates a lagrangian with terms linear or quadratic in the linear multiplet fields $C, \chi$ and $B_{\mu \nu}$. Before applying the Poincaré gauge fixing (3.6),

$$
\begin{aligned}
-\frac{1}{2} \operatorname{Im} \Upsilon^{2}= & -\frac{e}{8} \zeta^{2} \epsilon^{\mu \nu \rho \sigma} B_{\mu \nu} B_{\rho \sigma}+e \zeta C D-e \zeta \bar{\lambda} \chi+\frac{e}{4} \zeta \epsilon^{\mu \nu \rho \sigma} B_{\mu \nu} \widehat{\mathcal{F}}_{\rho \sigma} \\
& -\frac{i}{2} e \zeta C \bar{\psi}_{\mu} \gamma^{\mu} \gamma_{5} \lambda-\frac{1}{4} e \zeta \epsilon^{\mu \nu \rho \sigma} B_{\mu \nu} \bar{\psi}_{\rho} \gamma_{\sigma} \lambda+\text { derivative }
\end{aligned}
$$


Coupling the deformed super-Maxwell theory to new-minimal supergravity amounts to consider

$$
\mathcal{L}=\mathcal{L}_{\text {new min SG }}+\mathcal{L}_{\text {def Maxwell }}=\frac{3}{2}\left[L \ln \frac{L}{S \bar{S}}-L\right]_{D}-\frac{1}{2} \operatorname{Re}\left[\Upsilon^{2}\right]_{F}-\frac{\vartheta}{2} \operatorname{Im}\left[\Upsilon^{2}\right]_{F} .
$$

In components and with Poincaré gauge fixing (3.6), this lagrangian reads

$$
\begin{aligned}
e^{-1} \mathcal{L}= & \frac{1}{2 \kappa^{2}} R-\frac{1}{2 \kappa^{2}} \bar{\psi}_{\mu} \gamma^{\mu \nu \rho} \mathcal{D}_{\nu}^{(P)} \psi_{\rho} \\
& -\frac{\zeta^{2}}{2 \kappa^{4}}-\frac{1}{2} \bar{\lambda} \gamma^{a} \mathcal{D}_{a}^{(P)} \lambda-\frac{i \zeta}{2 \kappa^{2}} \bar{\psi}_{\mu} \gamma^{\mu} \gamma_{5}\left(\vartheta+i \gamma_{5}\right) \lambda-\frac{1}{8}\left(\bar{\psi}_{\mu} \gamma_{\nu} \lambda\right)\left(\bar{\psi}_{\rho} \gamma^{\rho \mu \nu} \lambda\right) \\
& +e^{-1} \mathcal{L}_{\text {aux. }} .
\end{aligned}
$$

The auxiliary lagrangian $\mathcal{L}_{\text {aux. }}$ includes the contributions of $\mathcal{B}_{a b}$, of $D$ and of the $\mathrm{U}(1)_{R}$ gauge field $A_{\mu}$. A first expression is

$$
\begin{aligned}
\mathcal{L}_{\text {aux. }}= & -\frac{3 e \kappa^{2}}{4} E_{\mu} E^{\mu}-3 e A_{\mu} E^{\mu}+\frac{3 i e}{4} A_{\mu} \bar{\lambda} \gamma^{\mu} \gamma_{5} \lambda+\frac{e}{2} D^{2}+\frac{e}{\kappa^{2}} \zeta \vartheta D \\
& -\frac{e}{4} \mathcal{B}^{a b} \mathcal{B}_{a b}-\frac{\vartheta}{8} e \epsilon^{\mu \nu \rho \sigma}\left(\mathcal{B}_{\mu \nu}+\bar{\psi}_{\mu} \gamma_{\nu} \lambda\right)\left(\mathcal{B}_{\rho \sigma}+\bar{\psi}_{\rho} \gamma_{\sigma} \lambda\right)-\frac{e}{4} \mathcal{B}_{c d} \bar{\psi}_{a} \gamma^{a c d} \lambda,
\end{aligned}
$$

where $E^{\mu}$ is given in eq. (4.2). The first two terms originate from the supergravity lagrangian, while all others from the Maxwell lagrangian.

Working from here on with $\zeta \neq 0$ and defining a new $\mathrm{U}(1)_{R}$ gauge field by generalising (3.13) as

$$
\frac{\zeta}{3} \widetilde{A}_{\mu}=A_{\mu}+\frac{\kappa^{2}}{4} E_{\mu}+\frac{\kappa^{2}}{16} i \bar{\lambda} \gamma_{\mu} \gamma_{5} \lambda, \quad \zeta \neq 0
$$

leads to a second expression:

$$
\begin{aligned}
\mathcal{L}_{\text {aux. }}= & -e \zeta \widetilde{A}_{\mu}\left(E^{\mu}-\frac{i}{4} \bar{\lambda} \gamma^{\mu} \gamma_{5} \lambda\right)-\frac{e}{4} \mathcal{B}^{a b} \mathcal{B}_{a b}-\frac{e}{4} \mathcal{B}_{c d} \bar{\psi}_{a} \gamma^{a c d} \lambda \\
& -\frac{\vartheta}{8} e \epsilon^{\mu \nu \rho \sigma}\left(\mathcal{B}_{\mu \nu}+\bar{\psi}_{\mu} \gamma_{\nu} \lambda\right)\left(\mathcal{B}_{\rho \sigma}+\bar{\psi}_{\rho} \gamma_{\sigma} \lambda\right) \\
& +\frac{3 e \kappa^{2}}{64}\left(\bar{\lambda} \gamma^{a} \gamma_{5} \lambda\right)\left(\bar{\lambda} \gamma_{a} \gamma_{5} \lambda\right)+\frac{e}{2} D^{2}+\frac{e}{\kappa^{2}} \zeta \vartheta D .
\end{aligned}
$$

The first contribution, which follows from the coupling $-3 e A_{\mu} E^{\mu}$ of eq. (4.17), indicates that even if $\mathcal{B}_{a b}$ has a two-derivative lagrangian in eq. (4.17), it does not propagate degrees of freedom. It is auxiliary, as in pure new-minimal supergravity, but it is not any longer a gauge field. It includes then six components, the number required by minimal supergravity. Using now expression (4.2) and integrating by parts, one finds an equivalent $\mathcal{L}_{\text {aux. }}$ in which $\mathcal{B}_{a b}$ has a simple algebraic field equation:

$$
\begin{aligned}
\mathcal{L}_{\text {aux. }}= & -\frac{e}{4} \mathcal{B}^{a b} \mathcal{B}_{a b}-\frac{\vartheta}{8} e \epsilon^{\mu \nu \rho \sigma} \mathcal{B}_{\mu \nu} \mathcal{B}_{\rho \sigma} \\
& -\frac{1}{4} e \epsilon^{\mu \nu \rho \sigma} \widehat{\widetilde{F}}_{\mu \nu} \mathcal{B}_{\rho \sigma}-\frac{1}{4} e \epsilon^{\mu \nu \rho \sigma} \widehat{\widetilde{F}}_{\mu \nu} \bar{\psi}_{\rho} \gamma_{\sigma} \lambda+\frac{e}{2} D^{2}+\frac{e}{\kappa^{2}} \zeta \vartheta D \\
& +\frac{i \zeta}{4} e \widetilde{A}_{\nu}\left(\frac{1}{\kappa^{2}} \bar{\psi}_{\mu} \gamma^{\mu \nu \rho} \gamma_{5} \psi_{\rho}+\bar{\lambda} \gamma^{\nu} \gamma_{5} \lambda\right)+\frac{\vartheta}{8} e \epsilon^{\mu \nu \rho \sigma}\left(\bar{\psi}_{\mu} \gamma_{\nu} \lambda\right)\left(\bar{\psi}_{\rho} \gamma_{\sigma} \lambda\right) \\
& +\frac{e}{4}\left(\bar{\psi}_{\mu} \gamma^{\mu \nu \rho} \lambda\right)\left(\bar{\psi}_{\nu} \gamma_{\rho} \lambda\right)+\frac{3 e \kappa^{2}}{64}\left(\bar{\lambda} \gamma^{a} \gamma_{5} \lambda\right)\left(\bar{\lambda} \gamma_{a} \gamma_{5} \lambda\right) .
\end{aligned}
$$


The above expression depends on the abelian field-strength of $\widetilde{A}_{\mu}$,

$$
\widehat{\widetilde{F}}_{\mu \nu}=\partial_{\mu} \widetilde{A}_{\nu}-\partial_{\nu} \widetilde{A}_{\mu}+\frac{1}{2} \bar{\psi}_{\mu} \gamma_{\nu}\left(i \gamma_{5}+\vartheta\right) \lambda-\frac{1}{2} \bar{\psi}_{\nu} \gamma_{\mu}\left(i \gamma_{5}+\vartheta\right) \lambda
$$

It is covariant under the supersymmetry variation

$$
\delta \widetilde{A}_{\mu}=-\frac{1}{2} \bar{\epsilon} \gamma_{\mu}\left(\vartheta+i \gamma_{5}\right) \lambda
$$

suggesting that the supersymmetric partner of $\widetilde{A}_{\mu}$ is $\widetilde{\lambda}=\left(\vartheta+i \gamma_{5}\right) \lambda$. Note that in eq. (4.20) the field strength $\widehat{\widetilde{F}}_{\mu \nu}$ constructed from the $\mathrm{U}(1)_{R}$ gauge field $A_{\mu}$ has a $\mathcal{B} \wedge \widehat{\widetilde{F}}$ (electriclike) interaction with the antisymmetric tensor while the original Maxwell field strength $\widehat{\mathcal{F}}_{\mu \nu}$ only appears in the (magnetic-like) combination $\mathcal{B}_{\mu \nu}=\zeta B_{\mu \nu}-\widehat{\mathcal{F}}_{\mu \nu}$, which is auxiliary (see eqs. (4.7) and (4.11)). As we will soon see, the emergence of the $\mathcal{B} \wedge \widehat{\widetilde{F}}$ coupling plays a crucial role in the integration of the auxiliary fields and an associated electric-magnetic duality transformation in the theory.

Eliminating $D$ leads to

$$
D=-\frac{\zeta \vartheta}{\kappa^{2}}
$$

implying the supersymmetry variations

$$
\delta \lambda=-\frac{\zeta}{2 \kappa^{2}}\left(1+i \vartheta \gamma_{5}\right) \epsilon+\text { linear }, \quad \delta \widetilde{\lambda}=-\frac{\zeta}{2 \kappa^{2}}\left(1+\vartheta^{2}\right) i \gamma_{5} \epsilon+\text { linear }
$$

while the cosmological constant becomes

$$
\Lambda=\frac{\zeta^{2}}{2 \kappa^{4}}\left(1+\vartheta^{2}\right)
$$

Finaly, eliminating $\mathcal{B}_{a b}$ leads to

$$
\mathcal{B}_{a b}=-\frac{1}{1+\vartheta^{2}}\left[\vartheta \widehat{\widetilde{F}}_{a b}+\frac{1}{2} \epsilon_{a b c d} \widehat{\widetilde{F}}^{c d}\right]
$$

It follows that

$$
\begin{aligned}
\mathcal{L}_{\text {aux. }}= & -\frac{e}{4\left(1+\vartheta^{2}\right)}\left[\widehat{\widetilde{F}}^{\mu \nu} \widehat{\widetilde{F}}_{\mu \nu}-\frac{\vartheta}{2} \epsilon^{\mu \nu \rho \sigma} \widehat{\widetilde{F}}_{\mu \nu} \widehat{\widetilde{F}}_{\rho \sigma}\right]-\frac{e \zeta^{2} \vartheta^{2}}{2 \kappa^{4}}-\frac{1}{4} e \epsilon^{\mu \nu \rho \sigma} \widehat{\widetilde{F}}_{\mu \nu} \bar{\psi}_{\rho} \gamma_{\sigma} \lambda \\
& +\frac{i \zeta}{4} e \widetilde{A}_{\nu}\left(\frac{1}{\kappa^{2}} \bar{\psi}_{\mu} \gamma^{\mu \nu \rho} \gamma_{5} \psi_{\rho}+\bar{\lambda} \gamma^{\nu} \gamma_{5} \lambda\right)+\frac{\vartheta}{8} e \epsilon^{\mu \nu \rho \sigma}\left(\bar{\psi}_{\mu} \gamma_{\nu} \lambda\right)\left(\bar{\psi}_{\rho} \gamma_{\sigma} \lambda\right) \\
& +\frac{e}{4}\left(\bar{\psi}_{\mu} \gamma^{\mu \nu \rho} \lambda\right)\left(\bar{\psi}_{\nu} \gamma_{\rho} \lambda\right)+\frac{3 e \kappa^{2}}{64}\left(\bar{\lambda} \gamma^{\mu} \gamma_{5} \lambda\right)\left(\bar{\lambda} \gamma_{\mu} \gamma_{5} \lambda\right),
\end{aligned}
$$


so that the complete lagrangian, upon elimination of $\mathcal{B}_{a b}$ and expressed in terms of $\widetilde{\lambda}$ and of the Poincaré spin connection $\omega_{\mu a b}(e)$ reads

$$
\begin{aligned}
\mathcal{L}= & \frac{1}{2 \kappa^{2}} e R-\frac{e}{2 \kappa^{2}} \bar{\psi}_{\mu} \gamma^{\mu \nu \rho} \mathcal{D}_{\nu}^{(P)} \psi_{\rho}-\frac{e \zeta^{2}}{2 \kappa^{4}}\left(1+\vartheta^{2}\right)-\frac{i e \zeta}{2 \kappa^{2}} \bar{\psi}_{\mu} \gamma^{\mu} \gamma_{5} \tilde{\lambda} \\
& -\frac{e}{4\left(1+\vartheta^{2}\right)}\left[\widehat{\widetilde{F}}^{\mu \nu} \widehat{\widetilde{F}}_{\mu \nu}-\frac{\vartheta}{2} \epsilon^{\mu \nu \rho \sigma} \widehat{\widetilde{F}}_{\mu \nu} \widehat{\widetilde{F}}_{\rho \sigma}-2 \overline{\widetilde{\lambda}} \gamma^{\mu} \mathcal{D}_{\mu}^{(P)} \widetilde{\lambda}\right] \\
& +\frac{i e \zeta}{4} \widetilde{A}_{\nu}\left(\frac{1}{\kappa^{2}} \bar{\psi}_{\mu} \gamma^{\mu \nu \rho} \gamma_{5} \psi_{\rho}+\frac{1}{1+\vartheta^{2}} \overline{\widetilde{\lambda}}^{\nu} \gamma_{5} \tilde{\lambda}\right) \\
& -\frac{e}{4\left(1+\vartheta^{2}\right)} \epsilon^{\mu \nu \rho \sigma}\left(\bar{\psi}_{\mu} \gamma_{\nu}\left(\vartheta-i \gamma_{5}\right) \widetilde{\lambda}\right)\left(\widehat{\widetilde{F}}_{\rho \sigma}-\frac{1}{2} \bar{\psi}_{\rho} \gamma_{\sigma} \tilde{\lambda}\right) \\
& +\frac{3 e \kappa^{2}}{64\left(1+\vartheta^{2}\right)^{2}}\left(\overline{\widetilde{\lambda}} \gamma^{\mu} \gamma_{5} \widetilde{\lambda}\right)\left(\overline{\widetilde{\lambda}} \gamma_{\mu} \gamma_{5} \widetilde{\lambda}\right)-\frac{e}{32\left(1+\vartheta^{2}\right)}\left(\overline{\widetilde{\lambda}} \gamma^{\mu \nu \rho} \widetilde{\lambda}\right)\left(\bar{\psi}_{\mu} \gamma_{\nu} \psi_{\rho}\right)+\mathcal{L}_{4, \mathrm{SG}},
\end{aligned}
$$

with field-strength

$$
\widehat{\widetilde{F}}_{\mu \nu}=\partial_{\mu} \widetilde{A}_{\nu}-\partial_{\nu} \widetilde{A}_{\mu}+\frac{1}{2} \bar{\psi}_{\mu} \gamma_{\nu} \widetilde{\lambda}-\frac{1}{2} \bar{\psi}_{\nu} \gamma_{\mu} \widetilde{\lambda}
$$

and with four-gravitino terms $\mathcal{L}_{4, \mathrm{SG}}$ given in eq. (3.17).

Since

$$
\epsilon^{\mu \nu \rho \sigma}\left(\widehat{\widetilde{F}}_{\mu \nu}-\bar{\psi}_{\mu} \gamma_{\nu} \widetilde{\lambda}\right)\left(\widehat{\widetilde{F}}_{\rho \sigma}-\bar{\psi}_{\rho} \gamma_{\sigma} \widetilde{\lambda}\right)
$$

is a derivative, one finally finds

$$
\begin{aligned}
\mathcal{L}= & \frac{e}{2 \kappa^{2}} R-\frac{e}{2 \kappa^{2}} \bar{\psi}_{\mu} \gamma^{\mu \nu \rho} \mathcal{D}_{\nu}^{(P)} \psi_{\rho}-\frac{e \zeta^{2}}{2 \kappa^{4}}\left(1+\vartheta^{2}\right)-\frac{i e \zeta}{2 \kappa^{2}} \bar{\psi}_{\mu} \gamma^{\mu} \gamma_{5} \widetilde{\lambda} \\
& -\frac{e}{4\left(1+\vartheta^{2}\right)}\left[\widehat{\widetilde{F}}^{\mu \nu} \widehat{\widetilde{F}}_{\mu \nu}-2 \overline{\widetilde{\lambda}} \gamma^{\mu} \mathcal{D}_{\mu}^{(P)} \tilde{\lambda}\right] \\
& +\frac{i e \zeta}{4} \widetilde{A}_{\nu}\left(\frac{1}{\kappa^{2}} \bar{\psi}_{\mu} \gamma^{\mu \nu \rho} \gamma_{5} \psi_{\rho}+\frac{1}{1+\vartheta^{2}} \overline{\widetilde{\lambda}} \gamma^{\nu} \gamma_{5} \tilde{\lambda}\right) \\
& +\frac{i e}{4\left(1+\vartheta^{2}\right)} \epsilon^{\mu \nu \rho \sigma}\left(\bar{\psi}_{\mu} \gamma_{\nu} \gamma_{5} \widetilde{\lambda}\right)\left(\widehat{\widetilde{F}}_{\rho \sigma}-\frac{1}{2} \bar{\psi}_{\rho} \gamma_{\sigma} \widetilde{\lambda}\right) \\
& +\frac{3 e \kappa^{2}}{64\left(1+\vartheta^{2}\right)^{2}}\left(\overline{\widetilde{\lambda}} \gamma^{\mu} \gamma_{5} \tilde{\lambda}\right)\left(\overline{\widetilde{\lambda}} \gamma_{\mu} \gamma_{5} \widetilde{\lambda}\right)-\frac{e}{32\left(1+\vartheta^{2}\right)}\left(\overline{\widetilde{\lambda}} \gamma^{\mu \nu \rho} \widetilde{\lambda}\right)\left(\bar{\psi}_{\mu} \gamma_{\nu} \psi_{\rho}\right) \\
& +\mathcal{L}_{4, \mathrm{SG}}+\text { derivative, }
\end{aligned}
$$

where the spin connection in the Poincare covariant derivative $\mathcal{D}_{\nu}^{(P)}$ is $\omega_{\mu a b}(e)$ of (3.15).

In the first line of the above expressions (4.28) and (4.30), the last two terms are such that the $\zeta^{2}$ contributions in their supersymmetry variations cancel. The second line is the super-Maxwell kinetic lagrangian with a gauge coupling $\sqrt{1+\vartheta^{2}}$. The third line implies that both the gravitino and the gaugino $\widetilde{\lambda}$ have $\mathrm{U}(1)$ charge $\zeta / 2$ in units of the $\mathrm{U}(1)$ gauge coupling of $\widetilde{A}_{\mu}$. The terms in the fourth line can also be seen in eqs. (4.7) or (4.11). Note that after canonical normalisation of the gauge and gaugino kinetic terms by a rescaling of $\widetilde{A}_{\mu}$ and $\widetilde{\lambda}$ with the factor $\sqrt{1+\vartheta^{2}}$, the super-Maxwell part of the lagrangian depends only on one parameter $q=\zeta \sqrt{1+\vartheta^{2}} / 2$ which amounts to the physical U(1) charge of the gaugino. In particular, the cosmological constant is given by

$$
\Lambda=\frac{2 q^{2}}{\kappa^{4}} \text {. }
$$


To exhibit electric-magnetic duality between the original $\widehat{\mathcal{F}}_{\mu \nu}$ (eq. (4.1)) and the dynamical $\widehat{\widetilde{F}}_{\mu \nu}$ (and their supersymmetry partners) and make the connection with the global supersymmetry case of section 2, we may introduce back a complex gauge coupling in front of $\Upsilon^{2}$, as in eq. (2.8): ${ }^{13}$

$$
\mathcal{L}_{\text {def Maxwell }}=-\frac{1}{2} \operatorname{Im}\left[\widetilde{\tau} \Upsilon^{2}\right]_{F}, \quad \quad \widetilde{\tau}=\vartheta+\frac{i}{\widetilde{g}^{2}} .
$$

Restoring the factors of $\widetilde{g}$ in the above analysis, it is easy to see that the gauge kinetic terms in the second line of eq. (4.28) read

$$
-\frac{e}{4} \operatorname{Im} \tau\left[\widehat{\widetilde{F}}^{\mu \nu} \widehat{\widetilde{F}}_{\mu \nu}-2 \overline{\widetilde{\lambda}}^{\mu} \mathcal{D}_{\mu}^{(P)} \widetilde{\lambda}\right]-\frac{1}{8} \operatorname{Re} \tau e \epsilon^{\mu \nu \rho \sigma} \widehat{\widetilde{F}}_{\mu \nu} \widehat{\widetilde{F}}_{\rho \sigma}
$$

with complex coupling

$$
\tau=-\frac{1}{\widetilde{\tau}} .
$$

Hence, the physical complex coupling of the propagating super-Maxwell fields is inverted with respect to the lagrangian coupling $\widetilde{\tau}$. This duality inversion is due to the presence in the first line of the auxiliary lagrangian (4.20) of terms quadratic in the auxiliary tensor $\mathcal{B}_{a b}$, as displayed in the solution (4.26). The above expression (4.30) indicates an "electric" theory, dual to the original deformed "magnetic" theory (4.3), as could be expected since the starting point was the $\zeta B_{\mu \nu}-\widehat{\mathcal{F}}_{\mu \nu}$ coupling of the original Maxwell field.

Note also the agreement with the globally supersymmetric deformed Maxwell theory case (2.9) and (2.11). Moreover the cosmological constant reads:

$$
\Lambda=\frac{\zeta^{2}}{2 \kappa^{4}} \frac{\overline{\tilde{\tau}} \tilde{\tau}}{\operatorname{Im} \tilde{\tau}}=\frac{\zeta^{2}}{2 \kappa^{4}} \frac{1}{\operatorname{Im} \tau},
$$

in agreement with $(2.13)$ for $\xi=0$. In the next section, we show that the "electric" supergravity theory (4.30), (4.33) corresponds to a standard gauging of the $R$-symmetry with the deformation parameter $\zeta$ being the coefficient of the Fayet-Iliopoulos term.

\section{On the Fayet-Iliopoulos term in supergravity}

In the new-minimal formulation, the super-Maxwell theory is obtained from the superconformal lagrangian

$$
\mathcal{L}_{\text {new-min, Max }}=\frac{3}{2}\left[L \ln \frac{L}{S \bar{S}}-L\right]_{D}-\frac{1}{2} \operatorname{Im}\left[\tau W^{2}\right]_{F} \quad ; \quad \tau=\theta+\frac{i}{g^{2}},
$$

where $W$ is the chiral spinor multiplet of the field-strength $\widehat{\mathcal{F}}_{\mu \nu}$ (up to a sign), defined in (4.1) and appendix B. With respect to our previous discussion, $W$ is the Maxwell submultiplet of $\Upsilon$ obtained by choosing the deformation parameter $\zeta=0$.

\footnotetext{
${ }^{13}$ It could also be understood as a background value of a holomorphic function of some neutral chiral matter superfields of the theory. In this case, of course, additional modifications of the lagrangian are needed which go beyond the scope of this paper.
} 
We have already seen in (4.13), and it is well-known, that $\operatorname{Im}\left[W^{2}\right]_{F}$ is a derivative irrelevant in the theory. The addition to $\mathcal{L}_{\text {new-min, Max }}$ of

$$
\mathcal{L}_{\mathrm{FI}}=\frac{3}{2} \xi[L V]_{D}
$$

with a real coefficient $\xi$, generates the Fayet-Iliopoulos term. This expression couples the compensating multiplet $L$ to the real $w=0$ multiplet $V$ of the Maxwell gauge field $\mathcal{A}_{\mu}$, corresponding to the chiral field-strength spinor multiplet $W$. It is invariant under gauge transformation $\delta V=\Lambda+\bar{\Lambda}(\Lambda$ is chiral with $w=0)$ since $[L(\Lambda+\bar{\Lambda})]_{D}$ is a derivative. Notice that one can also write

$$
\mathcal{L}=\mathcal{L}_{\text {new-min, Max }}+\mathcal{L}_{\mathrm{FI}}=\frac{3}{2}\left[L \ln L-L \ln \left(\bar{S} e^{-\xi V} S\right)-L\right]_{D}-\frac{1}{2} \operatorname{Im}\left[\tau W^{2}\right]_{F}
$$

and view the Fayet-Iliopoulos term as a gauging of the U(1) invariance of the (unphysical) chiral multiplet $S$.

Using the real multiplet tensor calculus, the product of the real gauge multiplet $V$ in Wess-Zumino gauge (B.15) with $L$ (3.2) has components

$$
L V=\left(0, \quad 0, \quad 0, \quad C \mathcal{A}_{a}, \quad C \lambda-\frac{i}{2} \gamma^{a} \gamma_{5} \chi \mathcal{A}_{a}, \quad C D+E^{a} \mathcal{A}_{a}-\bar{\chi} \lambda-\frac{1}{4} \bar{\chi} \gamma^{a} \gamma^{b} \psi_{a} \mathcal{A}_{b}\right)
$$

where the vector field $E_{a}$ is given in eq. (B.10) (or in eq. (3.8) after Poincaré gauge fixing). The residual invariance of the Wess-Zumino gauge is the bosonic gauge invariance $\delta \mathcal{A}_{\mu}=$ $\partial_{\mu} \alpha$. Inserting the $w=2$ multiplet $L V$ in the real action density formula gives

$$
\begin{aligned}
D & =e C D-e \bar{\chi} \lambda-\frac{i}{2} e C \bar{\psi}_{\mu} \gamma^{\mu} \gamma_{5} \lambda+e \mathcal{A}_{\mu}\left[E^{\mu}+\frac{1}{4} \bar{\psi}_{\nu}\left[\gamma^{\nu}, \gamma^{\mu}\right] \chi-\frac{1}{4} C \epsilon^{\mu \nu \rho \sigma} \bar{\psi}_{\nu} \gamma_{\rho} \psi_{\sigma}\right] \\
& =\frac{e}{2} \epsilon^{\mu \nu \rho \sigma} \mathcal{A}_{\mu} \partial_{\nu} B_{\rho \sigma}+e C D-e \bar{\chi} \lambda-\frac{i}{2} e C \bar{\psi}_{\mu} \gamma^{\mu} \gamma_{5} \lambda .
\end{aligned}
$$

After Poincaré gauge fixing (3.6),

$$
\frac{3}{2} e^{-1} \xi[L V]_{D}=\frac{3}{2} \xi\left[\frac{1}{\kappa^{2}} D-\frac{i}{2 \kappa^{2}} \bar{\psi}_{\mu} \gamma^{\mu} \gamma_{5} \lambda+\mathcal{A}_{\mu} E^{\mu}\right]+\frac{1}{2 \kappa^{2}} \frac{3}{4} i \xi \mathcal{A}_{\nu} \bar{\psi}_{\mu} \gamma^{\mu \nu \rho} \gamma_{5} \psi_{\rho}
$$

and the gravitino acquires a charge under the Maxwell U(1) symmetry.

Collecting the terms in (3.12) and (4.7) with $\zeta=0$ and combining them with (5.6), we find that the full lagrangian (5.3) reads

$$
\begin{aligned}
\mathcal{L}= & \frac{e}{2 \kappa^{2}}\left[R-\bar{\psi}_{\mu} \gamma^{\mu \nu \rho} \mathcal{D}_{\nu}^{(P)} \psi_{\rho}\right]-\frac{e}{4} \widehat{\mathcal{F}}_{\mu \nu} \widehat{\mathcal{F}}^{\mu \nu}-\frac{e}{2} \bar{\lambda} \gamma^{a} \mathcal{D}_{a}^{(P)} \lambda+\frac{e}{4} \bar{\psi}_{\rho} \gamma^{\mu \nu \rho} \lambda \widehat{\mathcal{F}}_{\mu \nu} \\
& +\frac{3}{4} i \xi \mathcal{A}_{\nu}\left[\frac{e}{2 \kappa^{2}} \bar{\psi}_{\mu} \gamma^{\mu \nu \rho} \gamma_{5} \psi_{\rho}+\frac{e}{2} \bar{\lambda} \gamma^{\nu} \gamma_{5} \lambda\right]-\frac{3 i e}{4 \kappa^{2}} \xi \bar{\psi}_{\mu} \gamma^{\mu} \gamma_{5} \lambda \\
& -\frac{e}{8}\left(\bar{\psi}_{\mu} \gamma_{\nu} \lambda\right)\left(\bar{\psi}_{\rho} \gamma^{\rho \mu \nu} \lambda\right)+\frac{3 e \kappa^{2}}{64}\left(\bar{\lambda} \gamma^{\mu} \gamma_{5} \lambda\right)\left(\bar{\lambda} \gamma_{\mu} \gamma_{5} \lambda\right)+\mathcal{L}_{\text {aux. }},
\end{aligned}
$$

where for simplicity we rescaled the gauge coupling away, and the auxiliary field lagrangian is

$$
\begin{aligned}
\mathcal{L}_{\text {aux. }}= & -3 e\left(E^{\mu}-\frac{i}{4} \bar{\lambda} \gamma^{\mu} \gamma_{5} \lambda\right)\left(A_{\mu}-\frac{\xi}{2} \mathcal{A}_{\mu}+\frac{\kappa^{2}}{4} E_{\mu}+\frac{i \kappa^{2}}{16} \bar{\lambda} \gamma_{\mu} \gamma_{5} \lambda\right) \\
& +\frac{e}{2} D^{2}+\frac{3 e}{2 \kappa^{2}} \xi D-3 e\left(\partial_{\mu} \phi\right)\left(E^{\mu}-\frac{i}{4 \kappa^{2}} \bar{\psi}_{\nu} \gamma^{\mu \nu \rho} \gamma_{5} \psi_{\rho}\right) .
\end{aligned}
$$


In the last term above, by following the original analysis of $[9,10]$, we introduced the Lagrange multiplier $\phi$ to impose the condition (3.10) with solution (3.8), so that $E^{\mu}$ is now an unconstrained vector. Under $\mathrm{U}(1)_{R}$ variations, $\delta_{R} A_{\mu}=\partial_{\mu} \lambda_{T}$ and also $\delta_{R} \phi=-\lambda_{T}$, leading to

$$
\delta_{R} \mathcal{L}_{\text {aux. }}=\frac{3}{2} i\left(\partial_{\mu} \lambda_{T}\right)\left[\frac{e}{2 \kappa^{2}} \bar{\psi}_{\mu} \gamma^{\mu \nu \rho} \gamma_{5} \psi_{\rho}+\frac{e}{2} \bar{\lambda} \gamma^{\nu} \gamma_{5} \lambda\right] .
$$

This variation cancels the $\mathrm{U}(1)_{R}$ variation of the Rarita-Schwinger and Dirac kinetic lagrangians. The theory has then local $\mathrm{U}(1)_{R}$ symmetry, as expected in the new-minimal formulation, and $A_{\mu}+\partial_{\mu} \phi$ is gauge invariant.

Redefining now the auxiliary $\mathrm{U}(1)_{R}$ gauge field as

$$
A_{\mu}^{\prime}=A_{\mu}-\frac{\xi}{2} \mathcal{A}_{\mu}+\frac{\kappa^{2}}{4} E^{\mu}+\frac{i \kappa^{2}}{16} \bar{\lambda} \gamma^{\mu} \gamma_{5} \lambda
$$

leads to

$$
\begin{aligned}
\mathcal{L}_{\text {aux. }}= & -3 e\left(E^{\mu}-\frac{i}{4} \bar{\lambda} \gamma^{\mu} \gamma_{5} \lambda\right)\left(A_{\mu}^{\prime}+\partial_{\mu} \phi\right)+\frac{e}{2} D^{2}+\frac{3 e}{2 \kappa^{2}} \xi D \\
& -\frac{3}{2} i\left(\partial_{\mu} \phi\right)\left[\frac{e}{2 \kappa^{2}} \bar{\psi}_{\mu} \gamma^{\mu \nu \rho} \gamma_{5} \psi_{\rho}+\frac{e}{2} \bar{\lambda} \gamma^{\nu} \gamma_{5} \lambda\right] .
\end{aligned}
$$

Solving next for $E^{\mu}, A_{\mu}^{\prime}$ and $D$ gives

$$
0=E^{\mu}-\frac{i}{4} \bar{\lambda} \gamma^{\mu} \gamma_{5} \lambda=A_{\mu}^{\prime}+\partial_{\mu} \phi, \quad D=-\frac{3}{2 \kappa^{2}} \xi ; \quad \mathcal{L}_{\text {aux. }}=-\frac{9 e}{8 \kappa^{4}} \xi^{2}
$$

while $\phi$ can be eliminated by a $\mathrm{U}(1)_{R}$ gauge choice.

From the second line of (5.7), one deduces that the $\mathrm{U}(1)$ charge of the fermions is $q=3 \xi / 4$. It follows that the final form of the lagrangian can be written as

$$
\begin{aligned}
\mathcal{L}= & \frac{e}{2 \kappa^{2}}\left[R-\bar{\psi}_{\mu} \gamma^{\mu \nu \rho} \mathcal{D}_{\nu}^{(P)} \psi_{\rho}\right]-\frac{e}{4} \widehat{\mathcal{F}}_{\mu \nu} \widehat{\mathcal{F}}^{\mu \nu}-\frac{e}{2} \bar{\lambda} \gamma^{a} \mathcal{D}_{a}^{(P)} \lambda+\frac{e}{4} \bar{\psi}_{\rho} \gamma^{\mu \nu \rho} \lambda \widehat{\mathcal{F}}_{\mu \nu} \\
& +i q \mathcal{A}_{\nu}\left[\frac{e}{2 \kappa^{2}} \bar{\psi}_{\mu} \gamma^{\mu \nu \rho} \gamma_{5} \psi_{\rho}+\frac{e}{2} \bar{\lambda} \gamma^{\nu} \gamma_{5} \lambda\right]-\frac{i e}{\kappa^{2}} q \bar{\psi}_{\mu} \gamma^{\mu} \gamma_{5} \lambda-\frac{2 e}{\kappa^{4}} q^{2} \\
& -\frac{e}{8}\left(\bar{\psi}_{\mu} \gamma_{\nu} \lambda\right)\left(\bar{\psi}_{\rho} \gamma^{\rho \mu \nu} \lambda\right)+\frac{3 e \kappa^{2}}{64}\left(\bar{\lambda} \gamma^{\mu} \gamma_{5} \lambda\right)\left(\bar{\lambda} \gamma_{\mu} \gamma_{5} \lambda\right)
\end{aligned}
$$

in terms of the spin connection $\omega_{\mu a b}(e, \psi)$ which still includes the contorsion tensor. ${ }^{14}$

The theory described by (5.13) is actually the model derived in a Poincaré formulation by Freedman in 1976 [12]. Indeed, it can be easily transformed from the new-minimal formulation (5.3) to the old-minimal, exhibiting the gauging of the $R$-symmetry under which the chiral compensator becomes charged. This can be done by introducing a real multiplet $U$ with $w=2$ and a chiral $T$ with $w=0$ (so that $T+\bar{T}$ is a real multiplet with $w=0)$. One can then rewrite (5.3) as

$$
\mathcal{L}=\frac{3}{2}\left[U \ln U-U \ln \left(\bar{S} e^{-\xi V} S\right)-U-(T+\bar{T}) U\right]_{D}-\frac{1}{2} \operatorname{Im}\left[\tau W^{2}\right]_{F},
$$

\footnotetext{
${ }^{14}$ See eqs. (3.14) and (3.16).
} 
where the field equation for the multiplet $T$ indicates that $U$ is a linear multiplet: $U=L$. On the other hand, solving for $U$, the field equation is

$$
\ln \left(\frac{U}{\bar{S} e^{-\xi V S}}\right)=T+\bar{T}
$$

and then

$$
\mathcal{L}=-\frac{3}{2}\left[\bar{S} e^{-\xi V} S e^{T+\bar{T}}\right]_{D}-\frac{1}{2} \operatorname{Im}\left[\tau W^{2}\right]_{F} .
$$

Defining the $w=1$ chiral compensator $S_{0}$ of the old-minimal supergravity as $S_{0}=e^{T} S$ leads to the old-minimal formulation of the theory

$$
\mathcal{L}_{\text {old-minimal }}=-\frac{3}{2}\left[\bar{S}_{0} e^{-\xi V} S_{0}\right]_{D}-\frac{1}{2} \operatorname{Im}\left[\tau W^{2}\right]_{F},
$$

where the Fayet-Iliopoulos term appears as a gauging of the $\mathrm{U}(1)_{R}$ symmetry acting on $S_{0}$.

It is now easy to check that (5.13), and thus (5.3) or equivalently (5.17), is identical to the lagrangian (4.30) obtained from the deformed Maxwell theory, upon normalising the kinetic terms and expressing it in terms of the single physical parameter of the Maxwell sector which is the fermion charge $q=3 \xi g / 4=\zeta \sqrt{1+\vartheta^{2}} / 2$ (upon putting back the gauge coupling in (5.13) according to (5.3)), implying the identification

$$
\zeta=\frac{3}{2} \xi
$$

where we restored the gauge coupling $\tilde{g}$ in (4.30) according to (2.8) and used eqs. (4.33) and (4.34). The equivalence of the two theories (5.3) and (4.15) with gauge coupling (4.32) thus suggests that the deformation in the Maxwell theory coupled to supergravity exhibits two properties:

- it corresponds to a "magnetic" Fayet-Iliopoulos term of the gauged $\mathrm{U}(1)_{R} R$ symmetry, in agreement with the result in global supersymmetry discussed in section 2, since their respective gauge couplings are related by the electro-magnetic duality relation (4.34);

- it provides a different realisation of the Freedman model on-shell (i.e. upon elimination of the auxiliary fields).

In the next section, we establish this connection by working out the explicit form of the electric-magnetic duality in supergravity.

\section{On the electric-magnetic duality}

The fact that the deformed and the standard Fayet-Iliopoulos theory provide different constructions of the same Freedman model suggests that electric-magnetic duality plays a role. This is also suggested by the fact that in both descriptions the physical Maxwell fields are not the same. The goal of this section is to study more precisely this role of electric-magnetic (EM) duality. 
In the deformed theory of section 4, before elimination of the auxiliary fields, two abelian gauge fields are present. The $\mathrm{U}(1)_{R}$ gauge field appears explicitly (without kinetic term) and the Maxwell gauge field only appears in $\mathcal{B}_{\mu \nu}$. Since no fields have Maxwell charge, one can expect that EM duality applies on the Maxwell field. The same situation occurs in the Fayet-Iliopoulos version of the theory described in section 5. To see this, a different form of $[L V]_{D}$ is useful. Starting with the second expression (5.5) and integrating by parts leads to

$$
\begin{aligned}
D & =\frac{e}{2} \epsilon^{\mu \nu \rho \sigma}\left(\partial_{\mu} \mathcal{A}_{\nu}\right) B_{\rho \sigma}-\frac{i}{2} e C \bar{\psi}_{\mu} \gamma^{\mu} \gamma_{5} \lambda+e C D-e \bar{\chi} \lambda \\
& =\frac{e}{4} \epsilon^{\mu \nu \rho \sigma} \widehat{\mathcal{F}}_{\mu \nu} B_{\rho \sigma}-\frac{e}{4} \epsilon^{\mu \nu \rho \sigma} B_{\mu \nu} \bar{\psi}_{\rho} \gamma_{\sigma} \lambda-\frac{i}{2} e C \bar{\psi}_{\mu} \gamma^{\mu} \gamma_{5} \lambda+e C D-e \bar{\chi} \lambda
\end{aligned}
$$

up to total derivatives. After Poincaré gauge fixing,

$$
[L V]_{D}=\frac{e}{4} \epsilon^{\mu \nu \rho \sigma} \widehat{\mathcal{F}}_{\mu \nu} B_{\rho \sigma}-\frac{e}{4} \epsilon^{\mu \nu \rho \sigma} B_{\mu \nu} \bar{\psi}_{\rho} \gamma_{\sigma} \lambda-\frac{i e}{2 \kappa^{2}} \bar{\psi}_{\mu} \gamma^{\mu} \gamma_{5} \lambda+\frac{e}{\kappa^{2}} D .
$$

Thus, the Maxwell gauge field only appears through its field-strength $\widehat{\mathcal{F}}_{\mu \nu}$ and there is a $B \wedge \widehat{\mathcal{F}}$ interaction. Applying EM duality should plausibly lead to a theory depending on $B-\widehat{\widetilde{\mathcal{F}}}$, in terms of the magnetic dual $\widehat{\widetilde{\mathcal{F}}}$ of the electric field-strength $\widehat{\mathcal{F}}$.

Consider the product $\Upsilon W$ of the Maxwell $W$ with the chiral spinor superfield having $L$ as submultiplet. Since $\Upsilon W$ is chiral with $w=3$, there is a superconformal action formula which gives

$$
\begin{aligned}
-\frac{1}{2} \operatorname{Im}[\Upsilon W]_{F} & =\frac{e}{8} \epsilon^{\mu \nu \rho \sigma}\left(\widehat{\mathcal{F}}_{\mu \nu}-\bar{\psi}_{\mu} \gamma_{\nu} \lambda\right)\left(\mathcal{B}_{\rho \sigma}+\bar{\psi}_{\rho} \gamma_{\sigma} \lambda\right)+\frac{e}{2} C D-\frac{e}{4} \bar{\lambda}\left(2 \chi+i C \gamma^{\mu} \gamma_{5} \psi_{\mu}\right) \\
& =\frac{e}{4} \epsilon^{\mu \nu \rho \sigma}\left(\partial_{\mu} \mathcal{A}_{\nu}\right) B_{\rho \sigma}+\frac{e}{2} C D-\frac{e}{4} \bar{\lambda}\left(2 \chi+i C \gamma^{\mu} \gamma_{5} \psi_{\mu}\right)
\end{aligned}
$$

omitting derivatives. Then, by comparing (6.1) with (6.3), one finds

$$
\operatorname{Im}[\Upsilon W]_{F}=-[L V]_{D}+\text { derivative } .
$$

In global Poincaré supersymmetry, the analogous statement is

$$
\int d^{2} \theta d^{2} \bar{\theta} L V=-\int d^{2} \theta \chi W+\int d^{2} \bar{\theta} \overline{\chi W}
$$

since $W_{\alpha}=-\frac{1}{4} \overline{D D} D_{\alpha} V$ and any real linear superfield can be written as $L=D \chi-\bar{D} \bar{\chi}$.

To discuss now EM duality, we need to introduce two new multiplets, unrelated to the ones used earlier. Firstly, a chiral spinor $\underline{\Upsilon}$, with components $\underline{\lambda}, \underline{C}, \underline{D}, \underline{\mathcal{B}}_{a b}, \underline{\chi}$. Secondly, a Maxwell multiplet $\widetilde{\mathcal{W}}$, with components $\widetilde{\lambda}, \widehat{\widehat{W}}_{\mu \nu}, \widetilde{D}$ and gauge field $\widetilde{W}_{\mu}$. Consider the lagrangian contribution (6.3):

$$
\begin{aligned}
-\frac{1}{2} \operatorname{Im}[\underline{\Upsilon \mathcal{W}}]_{F}= & \frac{e}{8} \epsilon^{\mu \nu \rho \sigma}\left(\widehat{\widetilde{W}}_{\mu \nu}-\bar{\psi}_{\mu} \gamma_{\nu} \widetilde{\lambda}\right)\left(\underline{\mathcal{B}}_{\rho \sigma}+\bar{\psi}_{\rho} \gamma_{\sigma} \underline{\lambda}\right)+\frac{e}{2} \underline{C} \widetilde{D}-\frac{e}{4} \overline{\widetilde{\lambda}}\left(2 \underline{\chi}+i \underline{C} \gamma^{\mu} \gamma_{5} \psi_{\mu}\right) \\
& + \text { derivative. }
\end{aligned}
$$


The field equations of the components of $\widetilde{\mathcal{W}}$ imply $\underline{C}=\underline{\chi}=0$ and

$$
\underline{\mathcal{B}}_{\rho \sigma}=-\partial_{\rho} \underline{A}_{\sigma}+\partial_{\sigma} \underline{A}_{\rho}-\frac{1}{2} \bar{\psi}_{\rho} \gamma_{\sigma} \underline{\lambda}+\frac{1}{2} \bar{\psi}_{\sigma} \gamma_{\rho} \underline{\lambda}
$$

for some gauge field $\underline{A}_{\mu}$. In other words, $\widetilde{\mathcal{W}}$ variation of the action implies that $\underline{\Upsilon}=$ $-\underline{W}$, a Maxwell multiplet. This also indicates that $\operatorname{Im}[\underline{W} \widetilde{\mathcal{W}}]_{F}$ is a derivative for any pair of Maxwell multiplets and that the contribution (6.6) is invariant under the gauge transformation

$$
\underline{\Upsilon} \quad \longrightarrow \quad \underline{\Upsilon}+\text { any Maxwell supermultiplet . }
$$

Super-Maxwell theory can then be written as

$$
\begin{aligned}
\mathcal{L}_{\text {super-Maxwell }} & =-\frac{1}{2} \operatorname{Im} \tau \operatorname{Re}\left[\underline{\Upsilon}^{2}\right]_{F}-\frac{1}{2} \operatorname{Re} \tau \operatorname{Im}\left[\underline{\Upsilon}^{2}\right]_{F}-\operatorname{Im}[\underline{\Upsilon} \widetilde{\mathcal{W}}]_{F} \\
& =-\frac{1}{2} \operatorname{Im}\left(\tau\left[\underline{\Upsilon}^{2}\right]_{F}\right)-\operatorname{Im}[\underline{\Upsilon} \widetilde{\mathcal{W}}]_{F}, \quad \tau=\theta+\frac{i}{g^{2}},
\end{aligned}
$$

which turns into

$$
\mathcal{L}_{E}=-\frac{1}{2 g^{2}} \operatorname{Re}\left[W^{2}\right]_{F}+\text { derivative }
$$

after the elimination of $\widetilde{\mathcal{W}}$ leading to $\underline{\Upsilon}=-W$. On the other hand, one could instead eliminate $\underline{\Upsilon}$ using its field equation

$$
\underline{\Upsilon}=-\frac{1}{\tau} \widetilde{\mathcal{W}}
$$

One then obtains another form of the theory

$$
\mathcal{L}_{M}=-\frac{1}{2} \operatorname{Im}\left(-\frac{1}{\tau}\left[\widetilde{\mathcal{W}}^{2}\right]_{F}\right)=-\frac{1}{2} \frac{1}{g^{2} \tau \bar{\tau}} \operatorname{Re}\left[\widetilde{\mathcal{W}}^{2}\right]_{F}+\text { derivative }
$$

which is the magnetic dual of super-Maxwell theory, with inverted complex coupling $\tilde{\tau}=$ $-1 / \tau$.

We next add the new-minimal supergravity (3.3) and the Fayet-Iliopoulos terms to the lagrangian (6.9):

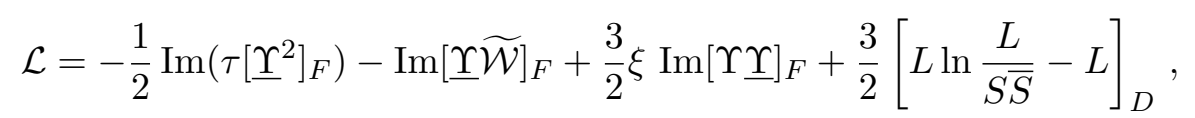

where $\Upsilon$ contains the compensator linear multiplet of the new-minimal formulation. Again, the field equation of $\widetilde{\mathcal{W}}$ is $\underline{\Upsilon}=-W$ and the term proportional to $\xi$ becomes the FayetIliopoulos contribution (5.2), leading to the action (5.3):

$$
\mathcal{L}_{E}=-\frac{1}{2} \operatorname{Im}\left[\tau W^{2}\right]_{F}+\frac{3}{2}\left[L \ln \frac{L}{\bar{S} e^{-\xi V S}}-L\right]_{D} .
$$

On the other hand, the field equation of $\underline{\Upsilon}$ gives now

$$
\Upsilon=-\frac{1}{\tau}\left(\widetilde{\mathcal{W}}-\frac{3}{2} \xi \Upsilon\right)=-\frac{1}{\tau}(\widetilde{\mathcal{W}}-\zeta \Upsilon),
$$


where we used the relation (5.18) between the Fayet-Iliopoulos parameter $\xi$ and the deformation parameter $\zeta$ used in section 4. Replacing $\Upsilon$ in (6.13), one obtains the magnetic dual form of the action:

$$
\mathcal{L}_{M}=-\frac{1}{2} \operatorname{Im}\left[-\frac{1}{\tau}(\widetilde{\mathcal{W}}-\zeta \Upsilon)^{2}\right]_{F}+\frac{3}{2}\left[L \ln \frac{L}{S \bar{S}}-L\right]_{D} .
$$

The last step is to observe that, if $\zeta \neq 0$ (and thus $\xi \neq 0$ ), the gauge transformation

$$
\begin{array}{lll}
\widetilde{\mathcal{W}} & \longrightarrow & \widetilde{\mathcal{W}}+X \\
\Upsilon & \longrightarrow & \Upsilon+\frac{1}{\zeta} X
\end{array}
$$

with $X$ an arbitrary Maxwell multiplet, leaves the compensating multiplet $L$ and thus the entire lagrangian invariant. The Maxwell multiplet $\widetilde{\mathcal{W}}$ can then be absorbed in the chiral spinor $\Upsilon$ and one can then write the magnetic lagrangian as

$$
\mathcal{L}_{M}=-\frac{1}{2} \operatorname{Im}\left[\tilde{\tau} \Upsilon^{2}\right]_{F}+\frac{3}{2}\left[L \ln \frac{L}{S \bar{S}}-L\right]_{D} ; \quad \tilde{\tau}=-\frac{1}{\tau} .
$$

The multiplet $\Upsilon$ in this last equation, which stands for $\zeta \Upsilon-\widetilde{\mathcal{W}}$, reduces to a Maxwell multiplet if $\zeta=0$; it is the chiral spinor multiplet used in the description of the deformed theory in section 4 . This completes the discussion of EM duality and proves the equivalence, by EM duality, of the deformed and the Fayet-Iliopoulos version of Freedman's model.

An alternative description of the $D$-deformation in supergravity and a derivation of the electromagnetic duality can be done using curved superspace techniques that we present in appendix C. In particular, we show that the deformation is dual to a magnetic FayetIliopoulos term, i.e. to the Freedman model, using similar steps as in section 2 that can be generalised to the case of supergravity, within the framework of curved conformal superspace.

\section{Concluding remarks}

In summary, we have studied in this work a deformation in $\mathcal{N}=1$ supersymmetry transformations corresponding to a shift of the real $D$-auxiliary field of a Maxwell multiplet by an imaginary constant, modifying the associated supersymmetric Bianchi identity by an integration constant. In global supersymmetry, the deformed theory is the electric-magnetic dual of a theory with a Fayet-Iliopoulos term with the deformation parameter mapped to its constant coefficient. An important property of the deformed theory is that (electrically) charged states cannot be added, implying that magnetic monopoles cannot exist for a U(1) with a Fayet-Iliopoulos term.

The coupling of the deformed theory to supergravity is achieved from the observation that the deformation can be seen as a background value of a linear multiplet which we identify with the linear compensating multiplet of the new-minimal formulation of $\mathcal{N}=1$ supergravity. Using the superconformal off-shell framework and introducing a general chiral spinor multiplet (contaning the field content of a linear and a Maxwell multiplets), we have 
shown that the deformed theory is again dual to a theory with Fayet-Iliopoulos term under electric-magnetic duality, generalising the result of global supersymmetry. In the absence of matter, the latter is the old Freedman model (1976) which gauges the $\mathrm{U}(1)_{R} R$-symmetry, under which the gravitino and gaugino are charged and there is a positive cosmological constant equal to twice the square of the charge in Planck units (see eq. (4.31)). In fact, the Freedman model can also be formulated in the new-minimal supergravity framework in terms of a chiral spinor multiplet containing the $\mathrm{U}(1)_{R}$ Maxwell multiplet together with the linear compensating multiplet. This formulation makes then the electro-magnetic duality between this model and the deformed theory manifest.

In the deformed theory, however, the Maxwell field is unobservable since it becomes part of a non-dynamical two-form field component of the chiral spinor multiplet. Upon integrating out the latter, the auxiliary gauge field of the superconformal $R$-symmetry becomes dynamical and the theory is shown to be on-shell, after elimination of auxiliary fields, equivalent to the Freedman model. This is consistent with the fact that the fermions (gravitino and gaugino) which are neutral under the orignal Maxwell field of the deformed theory lagrangian, as expected by the electric-magnetic duality, are charged under the $\mathrm{U}(1)_{R}$ symmetry.

The presence of a constant term in the supersymmetry variation of the gaugino, either due to the $\zeta$-deformation (4.24) or due to the non-trivial expectation value of the $D$ auxiliary field in the Freedman model (5.12), implies the existence of a 'unitary' gauge in which the gaugino vanishes and the lagrangian (4.30) or (5.13) simplifies considerably:

$$
e^{-1} \mathcal{L}_{\text {unit.gauge }}=\frac{1}{2 \kappa^{2}}\left[R-\bar{\psi}_{\mu} \gamma^{\mu \nu \rho} \mathcal{D}_{\nu}^{(P)} \psi_{\rho}\right]-\frac{1}{4} \mathcal{F}_{\mu \nu} \mathcal{F}^{\mu \nu}+i \frac{q}{2 \kappa^{2}} \mathcal{A}_{\nu} \bar{\psi}_{\mu} \gamma^{\mu \nu \rho} \gamma_{5} \psi_{\rho}-\frac{2}{\kappa^{4}} q^{2}
$$

Due to the positive cosmological constant, the background metric is de Sitter and the gravitino describes four propagating helicities despite the absence of an explicit mass term, since local supersymmetry is completely fixed. This phenomenon is similar to the case of the Volkov-Akulov model of non-linear supersymmetry [24-26] coupled to supergravity for vanishing gravitino mass-term [27-29], that can be obtained from the above expression by setting the gauge field $\mathcal{A}_{\mu}$ to zero.

One may worry about possible $\mathrm{U}(1)_{R}$ anomalies related to the existence of chiral fermions. Although there are already several studies in the literature [30-32], a careful investigation is needed for a firm conclusion, taking into account the de Sitter background in fermion propagators that also sets a non-vanishing fermion charge and the gravitinogaugino mixing in the lagrangian (4.30).

It would be interesting to generalise our analysis in the presence of matter chiral multiplets and field-dependent gauge couplings in both global and local supersymmetry. Another interesting question is to study the effects of the deformation in magnetic monopoles, which in principle can be described as charged states in the presence of a Fayet-Iliopoulos term in the dual theory. Finally, one could investigate the case of extended supergravity. In particular, the general non-trivial deformation of $\mathcal{N}=2$ supersymmetry transformations contains three parameters $[3,4]$ and is necessary for partial supersymmetry breaking $[2,3]$. It would be then interesting to work out the coupling of the deformed theory to $\mathcal{N}=2$ 
supergravity, ${ }^{15}$ and the possibility of partial supersymmetry breaking even in the absence of hypermultiplets.

\section{Acknowledgments}

We thank Friedemann Brandt and Sergei Kuzenko for correspondence. This work is supported in part by the Labex "Institut Lagrange de Paris" and in part by a CNRS PICS grant. I. A. and J.-P. D. wish to thank the University of Queensland, Brisbane for hospitality during part of this research work. H. J. is supported by the Swiss National Science Foundation. The work of G. T.-M. was supported by the Albert Einstein Center for Fundamental Physics, University of Bern, by the Australian Research Council (ARC) Future Fellowship FT180100353, and by the Capacity Building Package of the University of Queensland.

\section{A Conventions and some useful formulae}

We mostly follow the notations and conventions of ref. [13], with some exceptions. All spinors are Majorana and for the chiral projections we use $\lambda_{L}=P_{L} \lambda, \lambda_{R}=P_{R} \lambda$ and $\gamma_{5}$ instead of $\gamma_{*}$. We use the symbols $w, n$ to denote the Weyl and chiral weight, respectively.

- In local superconformal theory:

latin indices $a, b, c, \ldots$ denote the Lorentz (tangent space) indices, and they are raised and lowered by the flat metric $\eta_{a b}, \eta^{a b}=\operatorname{diag}(-+++)$.

Greek indices $\mu, \nu, \rho, \ldots$ are world indices, they are lowered by the metric $g_{\mu \nu}$, raised by its inverse $g^{\mu \nu}$.

Both types of indices are related using the vierbein field $e_{\mu}^{a}$ or its inverse $e_{a}^{\mu}$. In particular, we define $\partial_{a}=e_{a}^{\mu} \partial_{\mu}$.

- The antisymmetric symbols $\epsilon_{a b c d}$ and $\epsilon^{a b c d}$ are Lorentz tensor with numerical values $\pm 1,0, \epsilon_{0123}=-\epsilon^{0123}=1$. Then, $\epsilon^{\mu \nu \rho \sigma}$ and $\epsilon_{\mu \nu \rho \sigma}$ have Weyl weight $w=4$ and -4 , respectively, $e \epsilon^{\mu \nu \rho \sigma}$ and $e^{-1} \epsilon_{\mu \nu \rho \sigma}$ have $w=0$ and are numerical with value $0, \pm 1$. The matrices $\gamma^{a}$ are numerical while $\gamma^{\mu}=e_{a}^{\mu} \gamma^{a}$.

To eliminate the gauge fields of conformal boosts $f_{\mu}^{a}, \mathcal{S}$ supersymmetry $\phi_{\mu}$ and Lorentz symmetry (the spin connection $\omega_{\mu}^{a b}$ ), and obtain the $8_{B}+8_{F}$ Weyl multiplet of gauge fields, three invariant contraints are imposed on superconformal curvatures. They are respectively:

$$
e_{a}^{\mu} R_{\mu \nu}^{a b}(M)=-\frac{1}{2} \bar{\psi}_{a} \gamma_{\nu} R^{a b}(\mathcal{Q})-i e^{\rho b} \widetilde{R}_{\rho \nu}(T), \quad \gamma^{\mu} R_{\mu \nu}(\mathcal{Q})=0, \quad R_{\mu \nu}^{a}(P)=0
$$

and for the gauge field of Weyl symmetry, $b_{\mu}=0$ as part of the gauge-fixing to Poincaré symmetry. The third constraint leads then to eq. (3.14) while the first and second lead to

\footnotetext{
${ }^{15}$ Magnetic Fayet-Iliopoulos terms in $\mathcal{N}=2$ curved superspaces were described in [33, 34].
} 
the useful formula

$$
\begin{aligned}
& e_{a}^{\mu} f_{\mu}^{a}=-\frac{1}{12}\left[R+\frac{1}{2} \bar{\psi}_{\mu} \gamma^{\mu \nu \rho} \mathcal{D}_{\nu}^{(P)} \psi_{\rho}-\frac{3 i}{4} \bar{\psi}_{\mu} \gamma^{\mu \nu \rho} A_{\nu} \gamma_{5} \psi_{\rho}\right], \\
& \gamma^{\mu} \phi_{\mu}=-\frac{1}{12}\left[\gamma^{\mu}, \gamma^{\nu}\right] \mathcal{R}_{\mu \nu}(\psi), \quad\left[\gamma^{\mu}, \gamma^{\nu}\right] \phi_{\nu}=-\frac{1}{2} \gamma^{\mu \nu \rho} \mathcal{R}_{\nu \rho}(\psi)
\end{aligned}
$$

where

$$
\mathcal{R}_{\mu \nu}(\psi)=\mathcal{D}_{\mu}^{(P)} \psi_{\nu}-\mathcal{D}_{\nu}^{(P)} \psi_{\mu}-\frac{3}{2} i A_{\mu} \gamma_{5} \psi_{\nu}+\frac{3}{2} i A_{\nu} \gamma_{5} \psi_{\mu} .
$$

For a chiral multiplet $\mathcal{Z}=(Z, \chi, F)$ with weight $w=n=3$, the invariant $F$-density is given by

$$
e^{-1}[\mathcal{Z}]_{F}=F+\frac{1}{\sqrt{2}} \bar{\psi}_{\mu} \gamma^{\mu} \chi_{L}+\frac{1}{2} Z \bar{\psi}_{\mu} \gamma^{\mu \nu} \psi_{\nu R}, \quad \gamma^{\mu \nu}=\frac{1}{2}\left[\gamma^{\mu}, \gamma^{\nu}\right] .
$$

For a real multiplet $V=\left(C, \chi, H, B_{a}, \lambda, D\right)$ with weight $w=2, n=0$, the $D$-density is given by

$$
e^{-1}[V]_{D}=D+\square C-\frac{i}{2} \bar{\psi}_{\mu} \gamma^{\mu} \gamma_{5}\left(\lambda+\gamma^{b} D_{b} \chi\right)-\frac{1}{4} \bar{\psi}_{\mu} \gamma^{\mu \nu}\left(H \psi_{\nu L}+\bar{H} \psi_{\nu R}\right),
$$

where the superconformal d'alembertian is $\square=D^{a} D_{a}=e^{\mu a} D_{\mu} D_{a}, D_{a}=e_{a}^{\nu} D_{\nu}$, and we use $D_{\mu}$ for the covariant derivative of a given field with respect to the relevant local symmetry. The curvature constraints are used to prove the invariance of these densities.

Some rules of tensor calculus can be found in $[8,13]$.

Useful identities for $\gamma$-matrices include:

$$
\begin{aligned}
\gamma^{a b} \gamma_{5} & =\frac{i}{2} \epsilon^{a b c d} \gamma_{c d}, & \gamma^{a b c} \gamma_{5} & =i \epsilon^{a b c d} \gamma_{d}, \\
\gamma_{a} \gamma_{b c} & =\gamma_{a b c}+\eta_{a b} \gamma_{c}-\eta_{a c} \gamma_{b}, & \gamma_{a b} \gamma_{c} & =\gamma_{a b c}+\eta_{b c} \gamma_{a}-\eta_{a c} \gamma_{b} .
\end{aligned}
$$

Two useful four-fermion identities are

$$
\left(\bar{\lambda} \lambda_{L}\right)\left(\bar{\psi}_{\mu}\left[\gamma^{\mu}, \gamma^{\nu}\right] \psi_{\nu R}\right)+\text { c.c. }=2\left(\bar{\psi}_{\mu} \gamma_{\nu} \lambda\right)\left(\bar{\psi}_{\rho} \gamma^{\mu \nu \rho} \lambda\right)=2\left(\bar{\psi}_{\mu} \gamma_{\nu} \gamma_{5} \lambda\right)\left(\bar{\psi}_{\rho} \gamma^{\mu \nu \rho} \gamma_{5} \lambda\right)
$$

and

$$
\left(\bar{\lambda} \lambda_{L}\right)\left(\bar{\psi}_{\mu}\left[\gamma^{\mu}, \gamma^{\nu}\right] \psi_{\nu R}\right)-\text { c.c. }=2 i \epsilon^{\mu \nu \rho \sigma}\left(\bar{\psi}_{\mu} \gamma_{\nu} \lambda\right)\left(\bar{\psi}_{\rho} \gamma_{\sigma} \lambda\right)+2\left(\bar{\psi}_{\nu} \gamma^{\nu} \psi_{\mu}\right)\left(\bar{\lambda}^{\mu} \gamma_{5} \lambda\right) .
$$

\section{B The superconformal chiral spinor multiplet}

\section{B.1 Chiral spinor multiplet in the real field basis and its decomposition}

The lowest component of the chiral spinor multiplet is a spinor $\lambda$ with weights $w=n=3 / 2$. Its highest component is a second spinor $\chi$ with $w=5 / 2$ and $n=-3 / 2$. The eight bosonic fields are at the intermediate level with $w=2$ and $n=0$. They form a Lorentz chiral bispinor, four complex fields in Lorentz representation $(\mathbf{2}, \mathbf{1}) \times(\mathbf{2}, \mathbf{1})=(\mathbf{1}+\mathbf{3}, \mathbf{1})$. They admit an equivalent formulation in terms of an antisymmetric tensor $\mathcal{B}_{a b}$ and two real 
scalars $C$ and $D$. Since a detailed discussion of this superconformal multiplet does not seem to be available in the literature, this appendix provides the necessary information.

In this field basis, the $\mathcal{Q}$ (parameter $\epsilon$ ) and $\mathcal{S}$ (parameter $\eta$ ) supersymmetry variations $\operatorname{are}^{16}$

$$
\begin{aligned}
\delta \lambda & =-\frac{1}{2}\left(C-i D \gamma_{5}\right) \epsilon-\frac{1}{8}\left[\gamma^{a}, \gamma^{b}\right] \epsilon \mathcal{B}_{a b}, \\
\delta C & =\frac{i}{2} \bar{\epsilon} \gamma_{5} \chi \\
\delta D & =\frac{i}{2} \bar{\epsilon} \gamma_{5} \gamma^{a} D_{a} \lambda-\frac{1}{2} \bar{\epsilon} \chi \\
\delta \mathcal{B}_{a b} & =-\frac{i}{4} \bar{\epsilon}\left[\gamma_{a}, \gamma_{b}\right] \gamma_{5} \chi-\frac{1}{2} \bar{\epsilon} \gamma_{a} D_{b} \lambda+\frac{1}{2} \bar{\epsilon} \gamma_{b} D_{a} \lambda-\frac{1}{2} \bar{\eta}\left[\gamma_{a}, \gamma_{b}\right] \lambda \\
\delta \chi & =-\frac{i}{2} \gamma_{5} \gamma^{a} \epsilon D_{a} C-2 i \gamma_{5} \eta C+\frac{1}{2} E_{a} \gamma^{a} \epsilon .
\end{aligned}
$$

In the last variation,

$$
E^{d}=\frac{1}{2} \epsilon^{d a b c} D_{a} \mathcal{B}_{b c}
$$

with variation

$$
\delta E^{d}=-\frac{1}{4} \bar{\epsilon}\left[\gamma^{d}, \gamma^{a}\right] D_{a} \chi-\frac{3}{2} \bar{\eta} \gamma^{d} \chi
$$

The covariant derivatives are

$$
\begin{aligned}
D_{\mu} C= & \partial_{\mu} C-2 b_{\mu} C-\frac{i}{2} \bar{\psi}_{\mu} \gamma_{5} \chi \\
D_{\mu} \lambda= & \partial_{\mu} \lambda-\frac{3}{2}\left(b_{\mu}+i A_{\mu} \gamma_{5}\right) \lambda+\frac{1}{8} \omega_{\mu a b}\left[\gamma^{a}, \gamma^{b}\right] \lambda \\
& +\frac{1}{2} C \psi_{\mu}-\frac{i}{2} D \gamma_{5} \psi_{\mu}+\frac{1}{8}\left[\gamma^{a}, \gamma^{b}\right] \psi_{\mu} \mathcal{B}_{a b} \\
D_{\mu} \mathcal{B}_{b c}= & \partial_{\mu} \mathcal{B}_{b c}-2 b_{\mu} \mathcal{B}_{b c}-\omega_{\mu b}{ }^{d} \mathcal{B}_{d c}-\omega_{\mu c}{ }^{d} \mathcal{B}_{b d} \\
& +\frac{i}{4} \bar{\psi}_{\mu}\left[\gamma_{b}, \gamma_{c}\right] \gamma_{5} \chi+\frac{1}{2} \bar{\psi}_{\mu} \gamma_{b} D_{c} \lambda-\frac{1}{2} \bar{\psi}_{\mu} \gamma_{c} D_{b} \lambda+\frac{1}{2} \bar{\phi}_{\mu}\left[\gamma_{b}, \gamma_{c}\right] \lambda \\
D_{\mu} \chi= & \partial_{\mu} \chi-\frac{5}{2} b_{\mu} \chi+\frac{3}{2} i A_{\mu} \gamma_{5} \chi+\frac{1}{8} \omega_{\mu a b}\left[\gamma^{a}, \gamma^{b}\right] \chi \\
& +\frac{i}{2} \gamma_{5} \gamma^{a} \psi_{\mu} D_{a} C+2 i \gamma_{5} \phi_{\mu} C-\frac{1}{2} E_{a} \gamma^{a} \psi_{\mu} .
\end{aligned}
$$

The $8_{B}+8_{F}$ chiral spinor multiplet has two submultiplets with $4_{B}+4_{F}$ fields.

Maxwell multiplet. Firstly, choosing $C=\chi=0$ also requires $E^{a}=0$ and $\mathcal{B}_{a b}$ verifies then Bianchi identity $D_{[a} \mathcal{B}_{b c]}=0$. The fields $\lambda, D$ and $\mathcal{B}_{a b}=-\widehat{\mathcal{F}}_{a b}$ form a Maxwell multiplet with

$$
\widehat{\mathcal{F}}_{\mu \nu}=\partial_{\mu} \mathcal{A}_{\nu}-\partial_{\nu} \mathcal{A}_{\mu}+\frac{1}{2} \bar{\psi}_{\mu} \gamma_{\nu} \lambda-\frac{1}{2} \bar{\psi}_{\nu} \gamma_{\mu} \lambda
$$

which is indeed the covariant field-strength of a gauge field.

\footnotetext{
${ }^{16}$ In the conventions of ref. [13].
} 
The supersymmetry variations read

$$
\begin{aligned}
\delta \mathcal{A}_{\mu} & =-\frac{1}{2} \bar{\epsilon} \gamma_{\mu} \lambda, \\
\delta \widehat{\mathcal{F}}_{a b} & =\frac{1}{2} \bar{\epsilon} \gamma_{a} D_{b} \lambda-\frac{1}{2} \bar{\epsilon} \gamma_{b} D_{a} \lambda+\frac{1}{2} \bar{\eta}\left[\gamma_{a}, \gamma_{b}\right] \lambda, \\
\delta \lambda & =\frac{1}{8}\left[\gamma^{a}, \gamma^{b}\right] \epsilon \widehat{\mathcal{F}}_{a b}+\frac{i}{2} \gamma_{5} \epsilon D \\
\delta D & =\frac{i}{2} \bar{\epsilon} \gamma_{5} \gamma^{\mu} D_{\mu} \lambda .
\end{aligned}
$$

The covariant derivative in these expressions is

$$
\begin{aligned}
D_{\mu} \lambda & =\partial_{\mu} \lambda-\frac{3}{2}\left(b_{\mu}+i A_{\mu} \gamma_{5}\right) \lambda+\frac{1}{8} \omega_{\mu a b}\left[\gamma^{a}, \gamma^{b}\right] \lambda-\frac{1}{8}\left[\gamma^{a}, \gamma^{b}\right] \psi_{\mu} \widehat{\mathcal{F}}_{a b}-\frac{i}{2} D \gamma_{5} \psi_{\mu} \\
& \left.\equiv D_{\mu} \lambda\right|_{\text {Maxwell }}
\end{aligned}
$$

Returning to the components of the chiral spinor multiplet, another expression for $E^{\mu}=e_{d}^{\mu} E^{d}$ is

$$
\begin{aligned}
E^{\mu}= & \frac{1}{2} \epsilon^{\mu \nu \rho \sigma} \partial_{\nu} \mathcal{B}_{\rho \sigma}-\frac{1}{4} \bar{\psi}_{\nu}\left[\gamma^{\nu}, \gamma^{\mu}\right] \chi+\frac{1}{4} \epsilon^{\mu \nu \rho \sigma} \bar{\psi}_{\nu} \gamma_{\rho} \psi_{\sigma} C \\
& +\left.\frac{1}{2} \epsilon^{\mu \nu \rho \sigma} \bar{\psi}_{\nu} \gamma_{\rho} D_{\sigma} \lambda\right|_{\text {Maxwell }}+\frac{1}{4} \epsilon^{\mu \nu \rho \sigma} \bar{\phi}_{\nu}\left[\gamma_{\rho}, \gamma_{\sigma}\right] \lambda-\frac{1}{4} \epsilon^{\mu \nu \rho \sigma} \bar{\psi}_{\nu} \gamma^{\kappa} \psi_{\rho} \widehat{\mathcal{F}}_{\sigma \kappa} .
\end{aligned}
$$

In a Maxwell multiplet, the Bianchi identity leads to

$$
\frac{1}{2} \epsilon^{\mu \nu \rho \sigma} \partial_{\nu} \widehat{\mathcal{F}}_{\rho \sigma}=\epsilon^{\mu \nu \rho \sigma}\left(\left.\frac{1}{2} \bar{\psi}_{\nu} \gamma_{\rho} D_{\sigma} \lambda\right|_{\text {Maxwell }}+\frac{1}{4} \bar{\phi}_{\nu}\left[\gamma_{\rho}, \gamma_{\sigma}\right] \lambda-\frac{1}{4} \bar{\psi}_{\nu} \gamma^{a} \psi_{\rho} \widehat{\mathcal{F}}_{\sigma a}\right)
$$

which implies

$$
\begin{aligned}
E^{\mu} & =\frac{1}{2} \epsilon^{\mu \nu \rho \sigma} \partial_{\nu} B_{\rho \sigma}-\frac{1}{4} \bar{\psi}_{\nu}\left[\gamma^{\nu}, \gamma^{\mu}\right] \chi+\frac{1}{4} \epsilon^{\mu \nu \rho \sigma} \bar{\psi}_{\nu} \gamma_{\rho} \psi_{\sigma} C \\
B_{\mu \nu} & =\mathcal{B}_{\mu \nu}+\widehat{\mathcal{F}}_{\mu \nu}
\end{aligned}
$$

The Maxwell multiplet can be alternatively obtained from the real vector multiplet $V=\left(C, \chi, H, B_{a}, \lambda, D\right)$ with weight $w=n=0$. The gauge variation of $V$ is

$$
\delta_{g} V=\mathcal{V}(\mathcal{Z}+\overline{\mathcal{Z}})
$$

where $\mathcal{Z}=(Z, \varsigma, F)$ is a $w=0$ chiral multiplet and $\mathcal{V}(\mathcal{Z}+\overline{\mathcal{Z}})$ is the embedding of $\mathcal{Z}+\overline{\mathcal{Z}}$ in a vector multiplet with $w=0$. In components, the gauge variations read

$$
\begin{aligned}
\delta_{g} C & =Z+\bar{Z}, \quad \delta_{g} \chi=-\sqrt{2} i \gamma_{5} \varsigma, \quad \delta_{g} H=-2 F, \\
\delta_{g} B_{a} & =i D_{a}(Z-\bar{Z})=i \partial_{a}(Z-\bar{Z})-\frac{i}{\sqrt{2}} \bar{\psi}_{a} \gamma_{5} \varsigma=i \partial_{a}(Z-\bar{Z})+\frac{1}{2} \bar{\psi}_{a} \delta_{g} \chi, \\
\delta_{g} \lambda & =\delta_{g} D=0 .
\end{aligned}
$$

We can further define

$$
\mathcal{A}_{a}=e_{a}^{\mu} \mathcal{A}_{\mu}=B_{a}-\frac{1}{2} \bar{\psi}_{a} \chi
$$


which has the gauge variation $\delta_{g} \mathcal{A}_{\mu}=i \partial_{\mu}(Z-\bar{Z})$ required for a Maxwell gauge field. The supersymmetry variation of $\mathcal{A}_{\mu}$ is given by

$$
\delta \mathcal{A}_{\mu}=\frac{1}{2} \bar{\epsilon} \gamma_{\mu} \lambda
$$

as expected for the gauge field of a Maxwell multiplet in Wess-Zumino gauge with $\lambda$ and $D$ partner components in (B.6).

The gauge transformation in (B.12) enables us to impose the Wess-Zumino gauge condition on the vector multiplet:

$$
V_{\mathrm{WZ}}=\left(0, \quad 0, \quad 0, \quad \mathcal{A}_{a}+i \partial_{a}(Z-\bar{Z}), \quad \lambda, \quad D \quad\right)
$$

The supersymmetry variations of $\mathcal{A}_{\mu}, \lambda$ and $D$ are those of a Maxwell multiplet (B.6).

Linear multiplet. The second submultiplet is real and linear. In the chiral spinor multiplet, fields $C, \chi$ and $E^{a}$ transform into each others and they represent the algebra (the double variations close) if $D^{a} E_{a}=0$. Variations of the real linear multiplet are [7]

$$
\begin{aligned}
\delta C & =\frac{i}{2} \bar{\epsilon} \gamma_{5} \chi \\
\delta \chi & =-\frac{i}{2} \gamma_{5} \gamma^{a} \epsilon D_{a} C-2 i \gamma_{5} \eta C+\frac{1}{2} E_{a} \gamma^{a} \epsilon \\
\delta E^{a} & =-\frac{1}{4} \bar{\epsilon}\left[\gamma^{a}, \gamma^{b}\right] D_{b} \chi-\frac{3}{2} \bar{\eta} \gamma^{a} \chi
\end{aligned}
$$

with covariant derivatives

$$
\begin{aligned}
D_{\mu} C= & \partial_{\mu} C-2 b_{\mu} C-\frac{i}{2} \bar{\psi}_{\mu} \gamma_{5} \chi, \\
D_{\mu} \chi= & \partial_{\mu} \chi-\frac{5}{2} b_{\mu} \chi+\frac{3}{2} i A_{\mu} \gamma_{5} \chi+\frac{1}{8} \omega_{\mu a b}\left[\gamma^{a}, \gamma^{b}\right] \chi \\
& +\frac{i}{2} \gamma_{5} \gamma^{a} \psi_{\mu} D_{a} C+2 i \gamma_{5} \phi_{\mu} C-\frac{1}{2} E_{a} \gamma^{a} \psi_{\mu}
\end{aligned}
$$

and since one can rewrite $D^{a} E_{a}=0$ as

$$
0=\partial_{\mu}\left(e E^{\mu}+\frac{e}{4} \bar{\psi}_{\nu}\left[\gamma^{\nu}, \gamma^{\mu}\right] \chi-\frac{e}{4} \epsilon^{\mu \nu \rho \sigma} \bar{\psi}_{\rho} \gamma_{\sigma} \psi_{\nu} C\right)
$$

the solution is actually eq. (B.10) $[7,8]$.

The linear multiplet can be embedded in a real multiplet with weights $w=2, n=0$ as follows:

$$
L=\left(C, \quad \chi, \quad H=0, \quad B_{a}=-E_{a}, \quad \lambda=-\gamma^{b} D_{b} \chi, \quad D=-\square C\right), \quad D^{a} E_{a}=0 .
$$

Note that $[L]_{D}=0$ up to derivative.

We can in principle decompose the chiral spinor multiplet into two submultiplets: a Maxwell multiplet with fields $\lambda, D, \widehat{\mathcal{F}}_{a b}$ and a real linear multiplet with field $\chi, C, B_{a b}$ with

$$
\mathcal{B}_{a b}=B_{a b}-\widehat{\mathcal{F}}_{a b} \text {. }
$$


This decomposition is unstable under supersymmetry, but it is consistent. There are apparently two $E^{a}$ 's: in the chiral spinor multiplet $E_{a}$ is defined as the covariant field-strength of $\mathcal{B}_{a b}$ in eq. (B.2), while in the linear submultiplet, $E_{a}$ is defined by the constraint $D^{a} E_{a}=0$, eq. (B.18). The two definitions are consistent since they lead to the same solution (B.10) which only depends on the fields of the linear submultiplet.

\section{B.2 Chiral spinor multiplet in chiral multiplet basis}

Since the chiral spinor multiplet is chiral with $w=n=3 / 2$, the fields $\lambda, C, D, \mathcal{B}_{a b}$ and $\chi$ can be alternatively written as fields of a chiral multiplet, with components (spinor, bispinor, spinor):

$$
\begin{array}{ll}
w=n=3 / 2: & \lambda_{L}, \\
w=2, n=0: & -\frac{1}{\sqrt{2}}\left[(C-i D) \mathbb{I}+\frac{1}{8}\left(\mathcal{B}_{a b}+\frac{i}{2} \epsilon_{a b c d} \mathcal{B}^{c d}\right)\left[\gamma^{a}, \gamma^{b}\right]\right] P_{L}, \\
w=5 / 2, n=-3 / 2: & -2 i \chi_{L}-\gamma^{a} \mathcal{D}_{a} \lambda_{R} .
\end{array}
$$

These fields transform as expected for the components of a chiral multiplet with $w=n=$ $3 / 2$. This basis is especially useful for computing the square of the chiral spinor multiplet which has $w=n=3$ and can then be used to obtain a superconformal $F$-density action formula.

The square $\Upsilon^{2}=(Z, \varsigma, F)$ of the chiral spinor multiplet has components

$$
\begin{aligned}
w=n=3: & Z=\bar{\lambda} \lambda_{L}, \\
w=\frac{7}{2}, n=\frac{3}{2}: & \varsigma=-\sqrt{2}(C-i D) \lambda_{L}+\frac{\sqrt{2}}{4} \mathcal{B}_{a b}\left[\gamma^{a}, \gamma^{b}\right] \lambda_{L}, \\
w=4, n=0: & F=(C-i D)^{2}-\frac{i}{4} \epsilon^{a b c d} \mathcal{B}_{a b} \mathcal{B}_{c d}+\frac{1}{2} \mathcal{B}_{a b} \mathcal{B}^{a b}+2 \bar{\lambda} \gamma^{a} D_{a} \lambda_{R}+4 i \bar{\lambda} \chi_{L},
\end{aligned}
$$

where the covariant derivative is given in (B.4). This $w=n=3$ chiral multiplet leads to the superconformal $F$-density formula

$$
\begin{aligned}
e^{-1}\left[\Upsilon^{2}\right]_{F}= & (C-i D)^{2}+\frac{i}{4} \epsilon^{a b c d} \mathcal{B}_{a b} \mathcal{B}_{c d}+\frac{1}{2} \mathcal{B}_{a b} \mathcal{B}^{a b}+2 \bar{\lambda} \gamma^{a} D_{a} \lambda_{R}+4 i \bar{\lambda} \chi_{L} \\
& -(C-i D) \bar{\psi}_{\mu} \gamma^{\mu} \lambda_{L}+\frac{1}{4} \mathcal{B}_{a b} \bar{\psi}_{\mu} \gamma^{\mu}\left[\gamma^{a}, \gamma^{b}\right] \lambda_{L} \\
& +\frac{1}{4}\left(\bar{\lambda} \lambda_{L}\right)\left(\bar{\psi}_{\mu}\left[\gamma^{\mu}, \gamma^{\nu}\right] \psi_{\nu R}\right) .
\end{aligned}
$$

Then we can further compute its real part

$$
\begin{aligned}
e^{-1} \operatorname{Re}\left[\Upsilon^{2}\right]_{F}= & \frac{1}{2} \mathcal{B}_{a b} \mathcal{B}^{a b}-\frac{3 i}{2} \bar{\lambda} \gamma^{a} \gamma_{5} \lambda A_{a}+\frac{1}{2} \bar{\psi}_{c} \gamma^{c a b} \lambda \mathcal{B}_{a b}+C^{2}-D^{2}-C \bar{\psi}_{\mu} \gamma^{\mu} \lambda \\
& +\bar{\lambda} \gamma^{a} \mathcal{D}_{a}^{(P)} \lambda+\frac{1}{8}\left(\left(\bar{\lambda} \lambda_{L}\right)\left(\bar{\psi}_{\mu}\left[\gamma^{\mu}, \gamma^{\nu}\right] \psi_{\nu R}\right)+\text { c.c. }\right)+2 i \bar{\lambda} \gamma_{5} \chi
\end{aligned}
$$

and imaginary part

$$
\begin{aligned}
e^{-1} \operatorname{Im}\left[\Upsilon^{2}\right]_{F}= & -2 C D+\frac{1}{4} \epsilon^{a b c d}\left(\mathcal{B}_{a b}+\bar{\psi}_{a} \gamma_{b} \lambda\right)\left(\mathcal{B}_{c d}+\bar{\psi}_{c} \gamma_{d} \lambda\right) \\
& +i C \bar{\lambda} \gamma^{\mu} \gamma_{5} \psi_{\mu}+2 \bar{\lambda} \chi+\frac{i}{2} e^{-1} \partial_{\mu}\left(e e_{a}^{\mu} \bar{\lambda} \gamma^{a} \gamma_{5} \lambda\right)
\end{aligned}
$$


where we used the four-fermion identity (A.8), as well as the following relation ${ }^{17}$

$$
2 e \bar{\lambda} \gamma^{\mu} \gamma_{5} \mathcal{D}_{\mu}^{(P)} \lambda=\partial_{\mu}\left(e e_{a}^{\mu} \bar{\lambda} \gamma^{a} \gamma_{5} \lambda\right)+\frac{1}{2} e e_{a}^{\rho}\left(\bar{\psi}_{\mu} \gamma^{\mu} \psi_{\rho}\right)\left(\bar{\lambda} \gamma^{a} \gamma_{5} \lambda\right)+3 e \bar{\lambda} \gamma^{a} \gamma_{5} \lambda b_{a}
$$

\section{Deformed Maxwell theory in curved superspace}

In this appendix we describe a deformed vector multiplet in curved superspace. To make contact with the superconformal tensor calculus, it is natural to start by employing the conformal superspace approach to four-dimensional $\mathcal{N}=1$ conformal supergravity developed by Butter in [23] (see also the seminal work [35]). In this formalism, the superconformal group $\mathrm{SU}(2,2 \mid 1)$ is manifestly gauged in a curved superspace with covariant derivatives

$$
\nabla_{A}=\left(\nabla_{a}, \nabla_{\alpha}, \bar{\nabla}^{\dot{\alpha}}\right)=E_{A}^{M}\left(\partial_{M}-\mathbf{h}_{M^{\underline{I}}} \mathcal{M}_{\underline{I}}\right),
$$

where $E_{A}^{M}=E_{A}^{M}(x, \theta, \bar{\theta})$ is the superspace inverse vielbein ${ }^{18}$ while $\mathbf{h}_{M}{ }^{\underline{I}}=\mathbf{h}_{M} \underline{\underline{I}}(x, \theta, \bar{\theta})$ are gauge connections for all the superconformal generators except for translations and $\mathcal{Q}$ supersymmetry: $\mathcal{M}_{\underline{I}}=\left(M_{a b}, \mathbb{A}, \mathbb{D}, K_{a}, \mathcal{S}_{\alpha}, \overline{\mathcal{S}}^{\dot{\alpha}}\right) .{ }^{19}$ We refer the reader to [36-39] for reviews on supergravity in superspace while we refer to [23] for detail on conformal superspace that we will assume in this appendix. Note that the off-shell $8_{B}+8_{F}$ Weyl multiplet and the transformations of superconformal multiplets, can be derived following a $\theta=\bar{\theta}=0$ component-field projection (see refs. $[23,40]$ for more detail).

An abelian vector multiplet coupled to conformal supergravity is described by a superfield $\mathbf{W}_{\alpha}$ field strength which is a superconformal chiral $\left(\bar{\nabla}^{\dot{\alpha}} \mathbf{W}_{\alpha}=0\right)$ of weights $(3 / 2,1)$ satisfying the Bianchi identity

$$
\nabla^{\alpha} \mathbf{W}_{\alpha}=\bar{\nabla}_{\dot{\alpha}} \overline{\mathbf{W}}^{\dot{\alpha}}
$$

This is formally identical to the flat superspace one, eq. (2.1), as well as its solution which reads $\left(\nabla^{2}:=\nabla^{\alpha} \nabla_{\alpha}, \bar{\nabla}^{2}=\bar{\nabla}_{\dot{\alpha}} \bar{\nabla}^{\dot{\alpha}}\right)$

$$
\mathbf{W}_{\alpha}:=-\frac{1}{4} \bar{\nabla}^{2} \nabla_{\alpha} \mathbf{V}, \quad \overline{\mathbf{W}}^{\dot{\alpha}}:=-\frac{1}{4} \nabla^{2} \bar{\nabla}^{\dot{\alpha}} \mathbf{V},
$$

where $\mathbf{V}$ is a real scalar of weights $(0,0)$ and gauge transformation $\delta_{g} \mathbf{V}=(\boldsymbol{\Lambda}+\overline{\boldsymbol{\Lambda}})$, $\bar{\nabla}^{\dot{\alpha}} \boldsymbol{\Lambda}=0$. The Maxwell theory's action is based on the (anti-)chiral locally superconformal action principle (equivalent to the tensor calculus $F$-term density formula [23])

$$
S_{\text {Max }}=-\frac{1}{2} \operatorname{Im}\left[\tau \int d^{4} x d^{2} \theta \mathcal{E} \mathbf{W}^{2}\right], \quad \tau=\theta+\frac{i}{g^{2}} .
$$

\footnotetext{
${ }^{17} \mathrm{~A}$ useful equation to show (B.26) is $\partial_{\mu}\left(e e^{\mu}{ }_{a}\right)+3 e b_{a}+\omega_{\mu a b} e^{\mu b}+\frac{1}{2} e e^{\rho}{ }_{a} \bar{\psi}_{\mu} \gamma^{\mu} \psi_{\rho}=0$ using the curvature constraint $R_{\mu \nu}^{a}(P)=0$.

${ }^{18}$ Which includes the gauge fields $e_{\mu}^{a}(x)$ of space-time translations and the gravitino $\psi_{\mu}(x)$ of $\mathcal{Q}$ supersymmetry.

${ }^{19}$ The notation in this appendix differs from the rest of the paper and it adheres (up to some changes in nomenclature) to the one of [23,37], which is largely based on [36]. For example, we decompose fourdimensional Majorana spinors in chiral and anti-chiral parts. Compared to the flat superspace of section 2, following [23, 37], the spinor covariant derivatives satisfy the conjugation rule $\bar{\nabla}_{\dot{\alpha}}=\overline{\left(\nabla_{\alpha}\right)}$. Moreover, the normalisation of the $\mathrm{U}(1)_{R}$ generator $\mathbb{A}$ is $2 / 3$ of the generator $T$ used in [13] and earlier in this paper. Chiral weights in the two notations are related by $w_{\mathbb{A}}=2 / 3 n$ and the spinor covariant derivatives satisfy $\left[\mathbb{A}, \nabla_{\alpha}\right]=-i \nabla_{\alpha},\left[\mathbb{A}, \bar{\nabla}^{\dot{\alpha}}\right]=i \bar{\nabla}^{\dot{\alpha}}$.
} 
Next we want to analyse the possible deformation of the vector multiplet Bianchi identity in superspace. Note that, thanks to the algebra of $\nabla_{A}$, which for instance implies $\nabla_{\alpha} \nabla_{\beta} \nabla_{\gamma} \equiv 0$ as in the flat superspace [23], equalities $\nabla^{2}\left(\nabla^{\alpha} \mathbf{W}_{\alpha}\right)=0$ and $\bar{\nabla}^{2}\left(\nabla^{\alpha} \mathbf{W}_{\alpha}\right)=0$ hold. These properties rely on the weights of $\mathbf{W}_{\alpha}$ and $\nabla^{\alpha} \mathbf{W}_{\alpha}$. This implies that the following deformation of the Bianchi identity is consistent: ${ }^{20}$

$$
\nabla^{\alpha} \mathbf{W}_{\alpha}^{\text {def }}-\bar{\nabla}_{\dot{\alpha}} \overline{\mathbf{W}}^{\text {def } \dot{\alpha}}=-4 i \zeta \widehat{\mathbf{L}},
$$

where $\widehat{\mathbf{L}}$ is a real linear superfield of weights $(2,0)$ satisfying by definition

$$
\bar{\nabla}^{2} \widehat{\mathbf{L}}=\nabla^{2} \widehat{\mathbf{L}}=0 .
$$

We also define $\widehat{\mathbf{L}}$ as the compensating superconformal multiplet leading to new-minimal Poincaré supergravity. Then eq. (C.5) provides the curved superspace interpretation of the constraint (3.1) (the factor of $i$ is due to the different convention used in this appendix).

A solution of (C.5) is given by

$$
\mathbf{W}_{\alpha}^{\text {def }}=\mathbf{W}_{\alpha}-\zeta \widehat{\boldsymbol{\Upsilon}}_{\alpha}
$$

where $\mathbf{W}_{\alpha}$ is a regular undeformed vector multiplet field strength, see (C.2)-(C.3), while the chiral spinor superfield $\widehat{\Upsilon}_{\alpha}$ is such that

$$
\nabla^{\alpha} \widehat{\boldsymbol{\Upsilon}}_{\alpha}-\bar{\nabla}_{\dot{\alpha}} \overline{\boldsymbol{\Upsilon}}^{\dot{\alpha}}=4 i \widehat{\mathbf{L}}
$$

The solution (C.7) is gauge invariant under $\delta_{g} \widehat{\boldsymbol{\Upsilon}}_{\alpha}=\widehat{\mathbf{W}}_{\alpha}$ and $\delta_{g} \mathbf{W}_{\alpha}=\zeta \widehat{\mathbf{W}}_{\alpha}$ for some vector multiplet field strength $\widehat{\mathbf{W}}_{\alpha}$ and there is a gauge in which $\mathbf{W}_{\alpha}=0$. In other words, eq. (C.8) is not a constraint. It defines $\widehat{\mathbf{L}}$ for any $\widehat{\boldsymbol{\Upsilon}}_{\alpha}$.

The component fields of a $\mathbf{W}_{\alpha}^{\text {def }}$ coincide with the ones of a chiral spinor multiplet, $\left(\lambda, C, D, \mathcal{B}_{a b}, \chi\right)$, and simply arise as

$$
\begin{aligned}
& \lambda_{\alpha}:=\left.\mathbf{W}_{\alpha}^{\text {def }}\right|_{\theta=0}, \bar{\lambda}^{\dot{\alpha}}=\left.\overline{\mathbf{W}}^{\text {def } \dot{\alpha}}\right|_{\theta=0}, \quad D=-\left.\frac{1}{8}\left(\nabla^{\alpha} \mathbf{W}_{\alpha}^{\text {def }}+\bar{\nabla}_{\dot{\alpha}} \overline{\mathbf{W}}^{\operatorname{def} \dot{\alpha}}\right)\right|_{\theta=0}, \\
& \mathcal{B}_{a b}=\left.\mathbf{B}_{a b}\right|_{\theta=0}, \quad \mathbf{B}_{a b}:=\frac{\mathrm{i}}{2}\left(\left(\sigma_{a b}\right)^{\alpha \beta} \nabla_{\alpha} \mathbf{W}_{\beta}^{\text {def }}-\left(\bar{\sigma}_{a b}\right)_{\dot{\alpha} \dot{\beta}} \bar{\nabla}^{\dot{\alpha}} \overline{\mathbf{W}}^{\operatorname{def} \dot{\beta}}\right),
\end{aligned}
$$

while $\left(C, \chi_{\alpha}, \bar{\chi}^{\dot{\alpha}}\right)$, as well as the component field strength $E_{a}=\left.\frac{1}{3 !} \epsilon_{a b c d} \nabla^{b} \mathbf{B}^{c d}\right|_{\theta=0}$, are

$$
C=\left.\widehat{\mathbf{L}}\right|_{\theta=0}, \quad \chi_{\alpha}=\left.\nabla_{\alpha} \widehat{\mathbf{L}}\right|_{\theta=0}, \quad \bar{\chi}^{\dot{\alpha}}=\left.\bar{\nabla}^{\dot{\alpha}} \widehat{\mathbf{L}}\right|_{\theta=0}, \quad E_{a}=\left.\frac{1}{4}\left(\bar{\sigma}_{a}\right)^{\dot{\alpha} \alpha}\left[\nabla_{\alpha}, \bar{\nabla}_{\dot{\alpha}}\right] \widehat{\mathbf{L}}\right|_{\theta=0} .
$$

Local SU $(2,2 \mid 1)$ transformations of a chiral spinor multiplet can be straightforwardly derived from superspace and coincide (up to notation) with the ones presented in appendix B.

The gauge-fixing conditions of dilatations, $\mathcal{S}$-supersymmetry and special conformal transformations are as in eqs. (3.6), but their formulation in the curved superspace approach reads

$$
\widehat{\mathbf{L}}=\frac{1}{\kappa^{2}}, \quad \mathbf{B}_{M}=0,
$$

\footnotetext{
${ }^{20}$ Note that this constraint arises as the obstruction of the closure of the super two-form associated with an abelian vector multiplet induced by the closed super three-form of a linear multiplet compensator. This fits with the description of the abelian tensor hierarchy for $4 D, \mathcal{N}=1$ supersymmetry [41, 42].
} 
where $\mathbf{B}_{M}$ is the dilatation connection which is pure gauge for special (super)conformal transformations [23]. With this gauge fixing, the residual local transformations are superdiffeomorphisms, Lorentz, and $\mathrm{U}(1)_{R}$. The last two define the structure group of the off-shell new-minimal Poincaré supergravity geometry which is described by the covariant derivatives

$$
\mathcal{D}_{A}=E_{A}{ }^{M}\left(\partial_{M}-\frac{1}{2} \boldsymbol{\Omega}_{M}^{a b} M_{a b}-\mathbf{A}_{M} \mathbb{A}\right),
$$

with $\boldsymbol{\Omega}_{M}{ }^{a b}$ and $\mathbf{A}_{M}$ the Lorentz and $\mathrm{U}(1)_{R}$ connections, respectively. The geometry of $\mathcal{D}_{A}$, originally constructed in [43], can be derived by gauge fixing the $\nabla_{A}$ derivatives [23]. In the gauge (C.12), and in terms of the $\mathcal{D}_{A}$ derivatives, the Bianchi identity (C.5) turns into

$$
\mathcal{D}^{\alpha} \mathbf{W}_{\alpha}^{\text {def }}-\overline{\mathcal{D}}_{\dot{\alpha}} \overline{\mathbf{W}}^{\text {def }} \dot{\alpha}=-4 i \hat{\zeta}, \quad \hat{\zeta}:=\frac{\zeta}{\kappa^{2}},
$$

where $\hat{\zeta}$ is a constant, $\mathcal{D}_{A} \hat{\zeta}=\partial_{M} \hat{\zeta}=0$. This is the curved analog of the deformation of a Maxwell multiplet in flat superspace, eq. (2.4).

The superspace action for a deformed vector multiplet in the new-minimal supergravity background is then given by ${ }^{21}$

$$
S_{M}=-\frac{1}{2} \operatorname{Im}\left[\widetilde{\tau} \int d^{4} x d^{2} \theta \mathcal{E}\left(\mathbf{W}^{\text {def }}\right)^{2}\right], \quad \widetilde{\tau}=\frac{i}{\tilde{g}^{2}}+\vartheta .
$$

It is dual to a vector multiplet action with a Fayet-Iliopoulos term, precisely as shown in section 6. It is illustrative to show how the argument given in section 2 for the global case extends to curved superspace. Instead of expression (C.15), one starts from the action

$$
S=-\frac{1}{2} \operatorname{Im}\left[\widetilde{\tau} \int d^{4} x d^{2} \theta \mathcal{E} \boldsymbol{\Upsilon}^{2}\right]-\frac{i}{2} \int d^{4} x d^{2} \theta d^{2} \bar{\theta} E \mathbf{U}\left(\nabla^{\alpha} \boldsymbol{\Upsilon}_{\alpha}-\bar{\nabla}_{\dot{\alpha}} \overline{\mathbf{\Upsilon}}^{\dot{\alpha}}+4 i \zeta \widehat{\mathbf{L}}\right)
$$

where $\boldsymbol{\Upsilon}_{\alpha}$ is a chiral spinor superfield with weights $(3 / 2,1)$ and $\mathbf{U}$ is a zero-weight unconstrained real scalar. Eliminating $\mathbf{U}$ imposes the deformed Bianchi identity (C.5) on $\boldsymbol{\Upsilon}_{\alpha}$ and, with the identification $\boldsymbol{\Upsilon}_{\alpha}=\mathbf{W}_{\alpha}^{\text {def }}$, one obtains the "magnetic" action (C.15). Integrating by parts and redefining a full curved superspace as a (anti-)chiral superspace integral, ${ }^{22}$ the action (C.16) proves to be equivalent to

$$
S=-\frac{1}{2} \operatorname{Im}\left[\int d^{4} x d^{2} \theta \mathcal{E}\left(\widetilde{\tau} \Upsilon^{2}-\frac{1}{2} \Upsilon^{\alpha} \bar{\nabla}^{2} \nabla_{\alpha} \mathbf{U}\right)\right]+2 \zeta \int d^{4} x d^{2} \theta d^{2} \bar{\theta} E \widehat{\mathbf{L}} \mathbf{U}
$$

Eliminating the unconstrained $\mathbf{\Upsilon}$ leads firstly to

$$
\mathbf{\Upsilon}_{\alpha}=-\frac{1}{\widetilde{\tau}} \mathbf{W}_{\alpha}, \quad \mathbf{W}_{\alpha}:=-\frac{1}{4} \bar{\nabla}^{2} \nabla_{\alpha} \mathbf{U}
$$

\footnotetext{
${ }^{21}$ See [44] for a description in the old-minimal curved superspace geometry.

${ }^{22}$ Given a real lagrangian superfield $\mathscr{L}$ of conformal weights $(2,0)$, the full superspace integral is related to the (anti-)chiral superspace action as [23]

$$
\int d^{4} x d^{4} \theta E \mathscr{L}=-\frac{1}{4} \int d^{4} x d^{2} \theta \mathcal{E} \bar{\nabla}^{2} \mathscr{L}=-\frac{1}{4} \int d^{4} x d^{2} \bar{\theta} \overline{\mathcal{E}} \nabla^{2} \mathscr{L} .
$$

Local SU(2,2|1) invariants can be manipulated by using the rule for integration by parts [23].
} 
an undeformed abelian vector multiplet field strength, and secondly to the "electric" action

$$
S_{E}=-\frac{1}{2} \operatorname{Im}\left[\tau \int d^{4} x d^{2} \theta \mathcal{E} \mathbf{W}^{2}\right]+2 \zeta \int d^{4} x d^{2} \theta d^{2} \bar{\theta} E \widehat{\mathbf{L}} \mathbf{U}, \quad \tau=-\frac{1}{\widetilde{\tau}},
$$

where the second term is the curved superspace description for a standard Fayet-Iliopoulos term in new-minimal supergravity.

Open Access. This article is distributed under the terms of the Creative Commons Attribution License (CC-BY 4.0), which permits any use, distribution and reproduction in any medium, provided the original author(s) and source are credited.

\section{References}

[1] I. Antoniadis, H. Partouche and T.R. Taylor, Spontaneous breaking of $N=2$ global supersymmetry, Phys. Lett. B 372 (1996) 83 [hep-th/9512006] [INSPIRE].

[2] I. Antoniadis, J.-P. Derendinger and T. Maillard, Nonlinear $N=2$ supersymmetry, effective actions and moduli stabilization, Nucl. Phys. B 808 (2009) 53 [arXiv:0804.1738] [INSPIRE].

[3] I. Antoniadis, J.-P. Derendinger and C. Markou, Nonlinear $N=2$ global supersymmetry, JHEP 06 (2017) 052 [arXiv: 1703.08806] [INSPIRE].

[4] I. Antoniadis, H. Jiang and O. Lacombe, $N=2$ supersymmetry deformations, electromagnetic duality and Dirac-Born-Infeld actions, JHEP 07 (2019) 147 [arXiv: 1904.06339] [INSPIRE].

[5] S.M. Kuzenko, The Fayet-Iliopoulos term and nonlinear self-duality, Phys. Rev. D 81 (2010) 085036 [arXiv: 0911.5190] [INSPIRE].

[6] I. Antoniadis, H. Jiang and O. Lacombe, Note on supersymmetric Dirac-Born-Infeld action with Fayet-Iliopoulos term, JHEP 05 (2020) 111 [arXiv:1912.12627] [INSPIRE].

[7] B. de Wit and M. Roček, Improved tensor multiplets, Phys. Lett. B 109 (1982) 439 [INSPIRE].

[8] T. Kugo and S. Uehara, Conformal and Poincaré tensor calculi in $N=1$ supergravity, Nucl. Phys. B 226 (1983) 49 [INSPIRE].

[9] M.F. Sohnius and P.C. West, An alternative minimal off-shell version of $N=1$ supergravity, Phys. Lett. B 105 (1981) 353 [INSPIRE].

[10] M. Sohnius and P.C. West, The tensor calculus and matter coupling of the alternative minimal auxiliary field formulation of $N=1$ supergravity, Nucl. Phys. B 198 (1982) 493 [INSPIRE].

[11] S. Ferrara, L. Girardello, T. Kugo and A. Van Proeyen, Relation between different auxiliary field formulations of $N=1$ supergravity coupled to matter, Nucl. Phys. B 223 (1983) 191 [INSPIRE].

[12] D.Z. Freedman, Supergravity with axial gauge invariance, Phys. Rev. D 15 (1977) 1173 [INSPIRE].

[13] D.Z. Freedman and A.V. Proeyen, Supergravity, Cambridge University Press, Cambridge, U.K. (2009).

[14] F. Brandt, Supergravity with gauged second order symmetry, Phys. Lett. B 411 (1997) 79 [INSPIRE]. 
[15] F. Brandt, Deformed supergravity with local $R$ symmetry, hep-th/9704046 [INSPIRE].

[16] S. Ferrara, J. Wess and B. Zumino, Supergauge multiplets and superfields, Phys. Lett. B 51 (1974) 239 [INSPIRE].

[17] W. Siegel, Gauge spinor superfield as a scalar multiplet, Phys. Lett. B 85 (1979) 333 [INSPIRE].

[18] S.M. Kuzenko and S. Theisen, Supersymmetric duality rotations, JHEP 03 (2000) 034 [hep-th/0001068] [INSPIRE].

[19] S. Ferrara and P. van Nieuwenhuizen, The auxiliary fields of supergravity, Phys. Lett. B $\mathbf{7 4}$ (1978) 333 [INSPIRE].

[20] S. Ferrara and P. van Nieuwenhuizen, Tensor calculus for supergravity, Phys. Lett. B 76 (1978) 404 [INSPIRE].

[21] K.S. Stelle and P.C. West, Minimal auxiliary fields for supergravity, Phys. Lett. B 74 (1978) 330 [INSPIRE].

[22] K.S. Stelle and P.C. West, Tensor calculus for the vector multiplet coupled to supergravity, Phys. Lett. B 77 (1978) 376 [INSPIRE].

[23] D. Butter, $N=1$ conformal superspace in four dimensions, Annals Phys. 325 (2010) 1026 [arXiv: 0906.4399] [INSPIRE].

[24] D.V. Volkov and V.P. Akulov, Is the neutrino a Goldstone particle?, Phys. Lett. B 46 (1973) 109 [INSPIRE].

[25] M. Roček, Linearizing the Volkov-Akulov model, Phys. Rev. Lett. 41 (1978) 451 [INSPIRE].

[26] U. Lindström and M. Roček, Constrained local superfields, Phys. Rev. D 19 (1979) 2300 [INSPIRE].

[27] I. Antoniadis, E. Dudas, S. Ferrara and A. Sagnotti, The Volkov-Akulov-Starobinsky supergravity, Phys. Lett. B 733 (2014) 32 [arXiv:1403.3269] [INSPIRE].

[28] E. Dudas, S. Ferrara, A. Kehagias and A. Sagnotti, Properties of nilpotent supergravity, JHEP 09 (2015) 217 [arXiv:1507.07842] [INSPIRE].

[29] I. Antoniadis and C. Markou, The coupling of non-linear supersymmetry to supergravity, Eur. Phys. J. C $\mathbf{7 5}$ (2015) 582 [arXiv: 1508.06767] [INSPIRE].

[30] A.H. Chamseddine and H.K. Dreiner, Anomaly free gauged $R$ symmetry in local supersymmetry, Nucl. Phys. B 458 (1996) 65 [hep-ph/9504337] [INSPIRE].

[31] D.Z. Freedman and B. Körs, Kähler anomalies in supergravity and flux vacua, JHEP 11 (2006) 067 [hep-th/0509217] [inSPIRE].

[32] H. Elvang, D.Z. Freedman and B. Körs, Anomaly cancellation in supergravity with Fayet-Iliopoulos couplings, JHEP 11 (2006) 068 [hep-th/0606012] [INSPIRE].

[33] S.M. Kuzenko and G. Tartaglino-Mazzucchelli, Nilpotent chiral superfield in $N=2$ supergravity and partial rigid supersymmetry breaking, JHEP 03 (2016) 092 [arXiv: 1512.01964] [INSPIRE].

[34] S.M. Kuzenko and G. Tartaglino-Mazzucchelli, New nilpotent $N=2$ superfields, Phys. Rev. D 97 (2018) 026003 [arXiv: 1707.07390] [INSPIRE].

[35] T. Kugo and S. Uehara, $N=1$ superconformal tensor calculus: multiplets with external Lorentz indices and spinor derivative operators, Prog. Theor. Phys. 73 (1985) 235 [inSPIRE]. 
[36] J. Wess and J. Bagger, Supersymmetry and supergravity, Princeton University Press, Princeton, NJ, U.S.A. (1992).

[37] P. Binetruy, G. Girardi and R. Grimm, Supergravity couplings: a geometric formulation, Phys. Rept. 343 (2001) 255 [hep-th/0005225] [InSPIRE].

[38] S.J. Gates Jr., M.T. Grisaru, M. Roček and W. Siegel, Superspace or one thousand and one lessons in supersymmetry, Front. Phys. 58 (1983) 1 [hep-th/0108200] [INSPIRE].

[39] I.L. Buchbinder and S.M. Kuzenko, Ideas and methods of supersymmetry and supergravity, IOP, Bristol, U.K. (1995).

[40] T. Kugo, R. Yokokura and K. Yoshioka, Component versus superspace approaches to $D=4$, $N=1$ conformal supergravity, PTEP 2016 (2016) 073 [arXiv: 1602.04441] [INSPIRE].

[41] S.J. Gates Jr., Super p-form gauge superfields, Nucl. Phys. B 184 (1981) 381 [INSPIRE].

[42] R. Yokokura, Abelian tensor hierarchy and Chern-Simons actions in $4 D N=1$ conformal supergravity, JHEP 12 (2016) 092 [arXiv:1609.01111] [INSPIRE].

[43] M. Muller, Supergravity in U(1) superspace with a two form gauge potential, Nucl. Phys. B 264 (1986) 292 [INSPIRE].

[44] S.M. Kuzenko, On massive tensor multiplets, JHEP 01 (2005) 041 [hep-th/0412190] [INSPIRE]. 